\title{
Multiterminal Source Coding under Logarithmic Loss
}

\author{
Thomas A. Courtade* and Tsachy Weissman ${ }^{\dagger}$
}

November 1, 2018

\begin{abstract}
We consider the classical two-encoder multiterminal source coding problem where distortion is measured under logarithmic loss. We provide a single-letter description of the achievable rate distortion region for arbitrarily correlated sources with finite alphabets. In doing so, we also give the rate distortion region for the $m$-encoder CEO problem (also under logarithmic loss). Several applications and examples are given.
\end{abstract}

\section{Introduction}

A complete characterization of the achievable rate distortion region for the twoencoder source coding problem depicted in Figure 1 has remained an open problem for over three decades. Following tradition, we will refer to this two-encoder source coding network as the multiterminal source coding problem throughout this paper. Several special cases have been solved for general source alphabets and distortion measures:

- The lossless case where $D_{1}=0, D_{2}=0$. Slepian and Wolf solved this case in their seminal work 1 .

- The case where one source is recovered losslessly: i.e., $D_{1}=0, D_{2}=$ $D \max$. This case corresponds to the source coding with side information problem of Ahlswede-Körner-Wyner 2, 3].

- The Wyner-Ziv case [4] where $Y_{2}$ is available to the decoder as side information and $Y_{1}$ should be recovered with distortion at most $D_{1}$.

- The Berger-Yeung case (which subsumes the previous three cases) 5 where $D_{1}$ is arbitrary and $D_{2}=0$.

*Thomas Courtade is with the Department of Electrical Engineering, University of California, Los Angeles. Email: tacourta@ee.ucla.edu.

${ }^{\dagger}$ Tsachy Weissman is with the Department of Electrical Engineering, Stanford University. Email: tsachy@stanford.edu. 


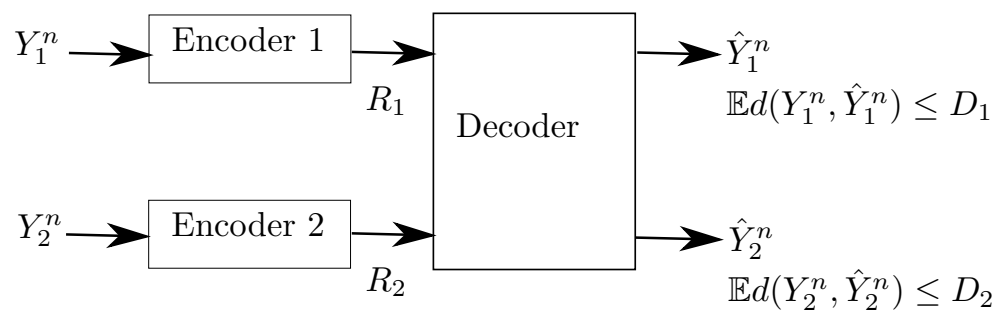

Figure 1: The multiterminal source coding network.

Despite the apparent progress, other seemingly fundamental cases, such as when $D_{1}$ is arbitrary and $D_{2}=D_{\max }$, remain unsolved except perhaps in very special cases.

Recently, the achievable rate distortion region for the quadratic Gaussian multiterminal source coding problem was given by Wagner, Tavildar, and Viswanath 6 . Until now, this was the only case for which the entire achievable rate distortion region was known. While this is a very important result, it is again a special case from a theoretical point of view: a specific choice of source distribution, and a specific choice of distortion measure.

In the present paper, we determine the achievable rate distortion region of the multiterminal source coding problem for arbitrarily correlated sources with finite alphabets. However, as in [6, we restrict our attention to a specific distortion measure.

At a high level, the roadmap for our argument is similar to that of [6]. In particular, both arguments couple the multiterminal source coding problem to a parametrized family of CEO problems. Then, the parameter in the CEO problem is "tuned" to yield the converse result. Despite this apparent similarity, the proofs in [6] rely heavily on the previously known Gaussian CEO results [7], the Gaussian one-helper results [8], and the calculus performed on the closed-form entropy expressions which arise from the Gaussian source assumption. In our case we do not have this luxury, and our CEO tuning argument essentially relies on an existence lemma to yield the converse result. The success of our approach is largely due to the fact that the distortion measure we consider admits a lower bound in the form of a conditional entropy, much like the quadratic distortion measure for Gaussian sources.

\subsection{Our Contributions}

In this paper, we give a single-letter characterization of the achievable rate distortion region for the multiterminal source coding problem under logarithmic loss. In the process of accomplishing this, we derive the achievable rate distortion region for the $m$-encoder CEO problem, also under logarithmic loss. In both settings, we obtain a stronger converse than is standard for rate distortion problems in the sense that augmenting the reproduction alphabet does not 
enlarge the rate distortion region. Notably, we make no assumptions on the source distributions, other than that the sources have finite alphabets. In both cases, the Berger-Tung inner bound on the rate distortion region is tight. To our knowledge, this constitutes the first time that the entire achievable rate distortion region has been described for general finite-alphabet sources under nontrivial distortion constraints.

\subsection{Organization}

This paper is organized as follows. In Section 2 we formally define the logarithmic loss function and the multiterminal source coding problem we consider. In Section 3 we define the CEO problem and give the rate distortion region under logarithmic loss. In Section 4 we return to the multiterminal source coding problem and derive the rate distortion region for the two-encoder setting. Also in Sections 3 and 4 , applications to estimation, horse racing, and list decoding are given. In Section 5 , we discuss connections between our results and the multiterminal source coding problem with arbitrary distortion measures. Section 6 delivers our concluding remarks and discusses directions for future work.

\section{Problem Definition}

Throughout this paper, we adopt notational conventions that are standard in the literature. Specifically, random variables are denoted by capital letters (e.g., $X)$ and their corresponding alphabets are denoted by corresponding calligraphic letters (e.g., $\mathcal{X})$. We abbreviate a sequence $\left(X_{1}, X_{2}, \ldots, X_{n}\right)$ of $n$ random variables by $X^{n}$, and we denote the interval $\left(X_{k}, X_{k+1}, \ldots, X_{j}\right)$ by $X_{k}^{j}$. If the lower index is equal to 1 , it will be omitted when there is no ambiguity (e.g., $X^{j} \triangleq X_{1}^{j}$ ). Frequently, random variables will appear with two subscripts (e.g., $Y_{i, j}$ ). In this case, we are referring to the $j^{\text {th }}$ instance of random variable $Y_{i}$. We overload our notation here slightly in that $Y_{i, 1}^{j}$ is often abbreviated as $Y_{i}^{j}$. However, our meaning will always be clear from context.

Let $\left\{\left(Y_{1, j}, Y_{2, j}\right)\right\}_{j=1}^{n}=\left(Y_{1}^{n}, Y_{2}^{n}\right)$ be a sequence of $n$ independent, identically distributed random variables with finite alphabets $\mathcal{Y}_{1}$ and $\mathcal{Y}_{2}$ respectively and joint $\operatorname{pmf} p\left(y_{1}, y_{2}\right)$. That is, $\left(Y_{1}^{n}, Y_{2}^{n}\right) \sim \prod_{i=1}^{n} p\left(y_{1, j}, y_{2, j}\right)$.

In this paper, we take the reproduction alphabet $\hat{\mathcal{Y}}_{i}$ to be equal to the set of probability distributions over the source alphabet $\mathcal{Y}_{i}$ for $i=1,2$. Thus, for a vector $\hat{Y}_{i}^{n} \in \hat{\mathcal{Y}}_{i}^{n}$, we will use the notation $\hat{Y}_{i, j}\left(y_{i}\right)$ to mean the $j^{\text {th }}$ coordinate $(1 \leq j \leq n)$ of $\hat{Y}_{i}^{n}$ (which is a probability distribution on $\mathcal{Y}_{i}$ ) evaluated for the

outcome $y_{i} \in \mathcal{Y}_{i}$. In other words, the decoder generates 'soft' estimates of the source sequences.

We will consider the logarithmic loss distortion measure defined as follows:

$$
d\left(y_{i}, \hat{y}_{i}\right)=\log \left(\frac{1}{\hat{y}_{i}\left(y_{i}\right)}\right)=D\left(1_{y_{i}}(y) \| \hat{y}_{i}(y)\right) \text { for } i=1,2 .
$$


In particular, $d\left(y_{i}, \hat{y}_{i}\right)$ is the relative entropy (i.e., Kullback-Leibler divergence) between the empirical distribution of the event $\left\{Y_{i}=y_{i}\right\}$ and the estimate $\hat{y}_{i}$. Using this definition for symbol-wise distortion, it is standard to define the distortion between sequences as

$$
d\left(y_{i}^{n}, \hat{y}_{i}^{n}\right)=\frac{1}{n} \sum_{j=1}^{n} d\left(y_{i, j}, \hat{y}_{i, j}\right) \text { for } i=1,2 .
$$

We point out that the logarithmic loss function is a widely used penalty function in the theory of learning and prediction (cf. [9, Chapter 9]). Further, it is a particularly natural loss criterion in settings where the reconstructions are allowed to be 'soft', rather than deterministic values. Surprisingly, since distributed learning and estimation problems are some of the most oft-cited applications of lossy multiterminal source coding, it does not appear to have been studied in this context until the recent work [10]. However, we note that this connection has been established previously for the single-encoder case in the study of the information bottleneck problem 11]. Beyond learning and prediction, a similar distortion measure has appeared before in the image processing literature 12. As we demonstrate through several examples, the logarithmic loss distortion measure has a variety of useful applications in the context of multiterminal source coding.

A rate distortion code (of blocklength $n$ ) consists of encoding functions:

$$
g_{i}^{(n)}: \mathcal{Y}_{i}^{n} \rightarrow\left\{1, \ldots, M_{i}^{(n)}\right\} \text { for } i=1,2
$$

and decoding functions

$$
\psi_{i}^{(n)}:\left\{1, \ldots, M_{1}^{(n)}\right\} \times\left\{1, \ldots, M_{2}^{(n)}\right\} \rightarrow \hat{\mathcal{Y}}_{i}^{n} \text { for } i=1,2 .
$$

A rate distortion vector $\left(R_{1}, R_{2}, D_{1}, D_{2}\right)$ is strict-sense achievable if there exists a blocklength $n$, encoding functions $g_{1}^{(n)}, g_{2}^{(n)}$ and a decoder $\left(\psi_{1}^{(n)}, \psi_{2}^{(n)}\right)$ such that

$$
\begin{aligned}
R_{i} & \geq \frac{1}{n} \log M_{i}^{(n)} \text { for } i=1,2 \\
D_{i} & \geq \mathbb{E} d\left(Y_{i}^{n}, \hat{Y}_{i}^{n}\right) \text { for } i=1,2 .
\end{aligned}
$$

Where

$$
\hat{Y}_{i}^{n}=\psi_{i}^{(n)}\left(g_{1}^{(n)}\left(Y_{1}^{n}\right), g_{2}^{(n)}\left(Y_{2}^{n}\right)\right) \text { for } i=1,2 .
$$

Definition 1. Let $\mathcal{R} \mathcal{D}^{\star}$ denote the set of strict-sense achievable rate distortion vectors and define the set of achievable rate distortion vectors to be its closure, $\overline{\mathcal{R D}}^{\star}$.

Our ultimate goal in the present paper is to give a single-letter characterization of the region $\overline{\mathcal{R D}}^{\star}$. However, in order to do this, we first consider an 
associated CEO problem. In this sense, the roadmap for our argument is similar to that of 6. Specifically, both arguments couple the multiterminal source coding problem to a parametrized family of CEO problems. Then, the parameter in the CEO problem is "tuned" to yield the converse result. Despite this apparent similarity, the proofs are quite different since the results in [6] depend heavily on the peculiarities of the Gaussian distribution.

\section{The CEO problem}

In order to attack the general multiterminal problem, we begin by studying the CEO problem (See 13 for an introduction.). To this end, let $\left\{\left(X_{j}, Y_{1, j}, Y_{2, j}\right)\right\}_{j=1}^{n}=\left(X^{n}, Y_{1}^{n}, Y_{2}^{n}\right)$ be a sequence of $n$ independent, identically distributed random variables distributed according to the joint pmf $p\left(x, y_{1}, y_{2}\right)=p(x) p\left(y_{1} \mid x\right) p\left(y_{2} \mid x\right)$. That is, $Y_{1} \leftrightarrow X \leftrightarrow Y_{2}$ form a Markov chain, in that order.

In this section, we consider the reproduction alphabet $\hat{\mathcal{X}}$ to be equal to the set of probability distributions over the source alphabet $\mathcal{X}$. As before, for a vector $\hat{X}^{n} \in \hat{\mathcal{X}}^{n}$, we will use the notation $\hat{X}_{j}(x)$ to mean the $j^{\text {th }}$ coordinate of $\hat{X}^{n}$ (which is a probability distribution on $\mathcal{X}$ ) evaluated for the outcome $x \in \mathcal{X}$. As in the rest of this paper, $d(\cdot, \cdot)$ is the logarithmic loss distortion measure.

A rate distortion CEO code (of blocklength $n$ ) consists of encoding functions:

$$
g_{i}^{(n)}: \mathcal{Y}_{i}^{n} \rightarrow\left\{1, \ldots, M_{i}^{(n)}\right\} \text { for } i=1,2
$$

and a decoding function

$$
\psi^{(n)}:\left\{1, \ldots, M_{1}^{(n)}\right\} \times\left\{1, \ldots, M_{2}^{(n)}\right\} \rightarrow \hat{\mathcal{X}}^{n} .
$$

A rate distortion vector $\left(R_{1}, R_{2}, D\right)$ is strict-sense achievable for the CEO problem if there exists a blocklength $n$, encoding functions $g_{1}^{(n)}, g_{2}^{(n)}$ and a decoder $\psi^{(n)}$ such that

$$
\begin{aligned}
R_{i} & \geq \frac{1}{n} \log M_{i}^{(n)} \text { for } i=1,2 \\
D & \geq \mathbb{E} d\left(X^{n}, \hat{X}^{n}\right) .
\end{aligned}
$$

Where

$$
\hat{X}^{n}=\psi^{(n)}\left(g_{1}^{(n)}\left(Y_{1}^{n}\right), g_{2}^{(n)}\left(Y_{2}^{n}\right)\right) .
$$

Definition 2. Let $\mathcal{R} \mathcal{D}_{C E O}^{\star}$ denote the set of strict-sense achievable rate distortion vectors and define the set of achievable rate distortion vectors to be its closure, $\overline{\mathcal{R D}}_{C E O}^{\star}$. 


\subsection{Inner Bound}

Definition 3. Let $\left(R_{1}, R_{2}, D\right) \in \mathcal{R} \mathcal{D}_{C E O}^{i}$ if and only if there exists a joint distribution of the form

$$
p\left(x, y_{1}, y_{2}\right) p\left(u_{1} \mid y_{1}, q\right) p\left(u_{2} \mid y_{2}, q\right) p(q)
$$

where $\left|\mathcal{U}_{1}\right| \leq\left|\mathcal{Y}_{1}\right|,\left|\mathcal{U}_{2}\right| \leq\left|\mathcal{Y}_{2}\right|$, and $|\mathcal{Q}| \leq 4$, which satisfies

$$
\begin{aligned}
R_{1} & \geq I\left(Y_{1} ; U_{1} \mid U_{2}, Q\right) \\
R_{2} & \geq I\left(Y_{2} ; U_{2} \mid U_{1}, Q\right) \\
R_{1}+R_{2} & \geq I\left(U_{1}, U_{2} ; Y_{1}, Y_{2} \mid Q\right) \\
D & \geq H\left(X \mid U_{1}, U_{2}, Q\right) .
\end{aligned}
$$

Theorem 1. $\mathcal{R D}_{C E O}^{i} \subseteq \overline{\mathcal{R D}}_{C E O}^{\star}$. That is, all rate distortion vectors $\left(R_{1}, R_{2}, D\right) \in \mathcal{R} \mathcal{D}_{C E O}^{i}$ are achievable.

Before proceeding with the proof, we cite the following variant of a wellknown inner bound:

Proposition 1 (Berger-Tung Inner Bound 14,15]). The rate distortion vector $\left(R_{1}, R_{2}, D\right)$ is achievable if

$$
\begin{aligned}
R_{1} & \geq I\left(U_{1} ; Y_{1} \mid U_{2}, Q\right) \\
R_{2} & \geq I\left(U_{2} ; Y_{2} \mid U_{1}, Q\right) \\
R_{1}+R_{2} & \geq I\left(U_{1}, U_{2} ; Y_{1}, Y_{2} \mid Q\right) \\
D & \geq \mathbb{E}\left[d\left(X, f\left(U_{1}, U_{2}, Q\right)\right]\right.
\end{aligned}
$$

for a joint distribution

$$
p(x) p\left(y_{1} \mid x\right) p\left(y_{2} \mid x\right) p\left(u_{1} \mid y_{1}, q\right) p\left(u_{2} \mid y_{2}, q\right) p(q)
$$

and reproduction function

$$
f: \mathcal{U}_{1} \times \mathcal{U}_{2} \times \mathcal{Q} \rightarrow \hat{\mathcal{X}}
$$

The proof of this proposition is a standard exercise in information theory, and is therefore omitted. The interested reader is directed to the text [16] for a modern, detailed treatment. The proposition follows from what is commonly called the Berger-Tung achievability scheme. In this encoding scheme, each encoder quantizes its observation $Y_{i}^{n}$ to a codeword $U_{i}^{n}$, such that the empirical distribution of the entries in $\left(Y_{i}^{n}, U_{i}^{n}\right)$ is very close to the true distribution $p\left(y_{i}, u_{i}\right)$. In order to communicate their respective quantizations to the decoder, the encoders essentially perform Slepian-Wolf coding. For this reason, the Berger-Tung achievability scheme is also referred to as a "quantize-and-bin" coding scheme. 
Proof of Theorem 1. Given Proposition 1, the proof of Theorem 1 is immediate. Indeed, if we apply Proposition 1 with the reproduction function $f\left(U_{1}, U_{2}, Q\right) \triangleq$ $\operatorname{Pr}\left[X=x \mid U_{1}, U_{2}, Q\right]$, we note that

$$
\mathbb{E}\left[d\left(X, f\left(U_{1}, U_{2}, Q\right)\right]=H\left(X \mid U_{1}, U_{2}, Q\right),\right.
$$

which yields the desired result.

Thus, from the proof of Theorem 1 , we see that our inner bound $\mathcal{R} \mathcal{D}_{C E O}^{i}$ simply corresponds to a specialization of the general Berger-Tung inner bound to the case of logarithmic loss.

\subsection{A Matching Outer Bound}

A particularly useful property of the logarithmic loss distortion measure is that the expected distortion is lower-bounded by a conditional entropy. A similar property is enjoyed by Gaussian random variables under quadratic distortion. In particular, if $G$ is Gaussian, and $\hat{G}$ is such that $\mathbb{E}(\hat{G}-G)^{2} \leq D$, then $\frac{1}{2} \log (2 \pi e) D \geq h(G \mid \hat{G})$. The case for logarithmic loss is similar, and we state it formally in the following lemma which is crucial in the proof of the converse.

Lemma 1. Let $Z=\left(g_{1}^{(n)}\left(Y_{1}^{n}\right), g_{2}^{(n)}\left(Y_{2}^{n}\right)\right)$ be the argument of the reproduction function $\psi^{(n)}$. Then $n \mathbb{E} d\left(X^{n}, \hat{X}^{n}\right) \geq H\left(X^{n} \mid Z\right)$.

Proof. By definition of the reproduction alphabet, we can consider the reproduction $\hat{X}^{n}$ to be a probability distribution on $\mathcal{X}^{n}$ conditioned on the argument $Z$. In particular, if $\hat{x}^{n}=\psi^{(n)}(z)$, define $s\left(x^{n} \mid z\right) \triangleq \prod_{j=1}^{n} \hat{x}_{j}\left(x_{j}\right)$. It is readily verified that $s$ is a probability measure on $\mathcal{X}^{n}$. Then, we obtain the following lower bound on the expected distortion conditioned on $Z=z$ :

$$
\begin{aligned}
\mathbb{E}\left[d\left(X^{n}, \hat{X}^{n}\right) \mid Z=z\right] & =\frac{1}{n} \sum_{j=1}^{n} \sum_{x^{n} \in \mathcal{X}^{n}} p\left(x^{n} \mid z\right) \log \left(\frac{1}{\hat{x}_{j}\left(x_{j}\right)}\right) \\
& =\frac{1}{n} \sum_{x^{n} \in \mathcal{X}^{n}} p\left(x^{n} \mid z\right) \sum_{j=1}^{n} \log \left(\frac{1}{\hat{x}_{j}\left(x_{j}\right)}\right) \\
& =\frac{1}{n} \sum_{x^{n} \in \mathcal{X}^{n}} p\left(x^{n} \mid z\right) \log \left(\frac{1}{s\left(x^{n} \mid z\right)}\right) \\
& =\frac{1}{n} \sum_{x^{n} \in \mathcal{X}^{n}} p\left(x^{n} \mid z\right) \log \left(\frac{p\left(x^{n} \mid z\right)}{s\left(x^{n} \mid z\right)}\right)+\frac{1}{n} H\left(X^{n} \mid Z=z\right) \\
& =\frac{1}{n} D\left(p\left(x^{n} \mid z\right) \| s\left(x^{n} \mid z\right)\right)+\frac{1}{n} H\left(X^{n} \mid Z=z\right) \\
& \geq \frac{1}{n} H\left(X^{n} \mid Z=z\right),
\end{aligned}
$$

where $p\left(x^{n} \mid z\right)=\operatorname{Pr}\left(X^{n}=x^{n} \mid Z=z\right)$ is the true conditional distribution. Averaging both sides over all values of $Z$, we obtain the desired result. 
Definition 4. Let $\left(R_{1}, R_{2}, D\right) \in \mathcal{R D}_{C E O}^{o}$ if and only if there exists a joint distribution of the form

$$
p(x) p\left(y_{1} \mid x\right) p\left(y_{2} \mid x\right) p\left(u_{1} \mid y_{1}, q\right) p\left(u_{2} \mid y_{2}, q\right) p(q),
$$

which satisfies

$$
\left.\begin{array}{rl}
R_{1} & \geq I\left(Y_{1} ; U_{1} \mid X, Q\right)+H\left(X \mid U_{2}, Q\right)-D \\
R_{2} & \geq I\left(Y_{2} ; U_{2} \mid X, Q\right)+H\left(X \mid U_{1}, Q\right)-D \\
R_{1}+R_{2} & \geq I\left(U_{1} ; Y_{1} \mid X, Q\right)+I\left(U_{2} ; Y_{2} \mid X, Q\right)+H(X)-D \\
D & \geq H\left(X \mid U_{1}, U_{2}, Q\right) .
\end{array}\right\}
$$

Theorem 2. If $\left(R_{1}, R_{2}, D\right)$ is strict-sense achievable for the CEO problem, then $\left(R_{1}, R_{2}, D\right) \in \mathcal{R D}_{C E O}^{o}$.

Proof. Suppose the point $\left(R_{1}, R_{2}, D\right)$ is strict-sense achievable. Let $A$ be a nonempty subset of $\{1,2\}$, and let $F_{i}=g_{i}^{(n)}\left(Y_{i}^{n}\right)$ be the message sent by encoder $i \in\{1,2\}$. Define $U_{i, j} \triangleq\left(F_{i}, Y_{i}^{j-1}\right)$ and $Q_{j} \triangleq\left(X^{j-1}, X_{j+1}^{n}\right)=X^{n} \backslash X_{j}$. To simplify notation, let $Y_{A}=\cup_{i \in A} Y_{i}$ (similarly for $U_{A}$ and $F_{A}$ ).

With these notations established, we have the following string of inequalities:

$$
\begin{aligned}
n \sum_{i \in A} R_{i} & \geq \sum_{i \in A} H\left(F_{i}\right) \\
& \geq H\left(F_{A}\right) \\
& \geq I\left(Y_{A}^{n} ; F_{A} \mid F_{A^{c}}\right) \\
& =I\left(X^{n}, Y_{A}^{n} ; F_{A} \mid F_{A^{c}}\right) \\
& =I\left(X^{n} ; F_{A} \mid F_{A^{c}}\right)+\sum_{i \in A} I\left(F_{i} ; Y_{i}^{n} \mid X^{n}\right) \\
& =H\left(X^{n} \mid F_{A^{c}}\right)-H\left(X^{n} \mid F_{1}, F_{2}\right)+\sum_{i \in A} \sum_{j=1}^{n} I\left(Y_{i, j} ; F_{i} \mid X^{n}, Y_{i}^{j-1}\right) \\
& \geq H\left(X^{n} \mid F_{A^{c}}\right)+\sum_{i \in A} \sum_{j=1}^{n} I\left(Y_{i, j} ; F_{i} \mid X^{n}, Y_{i}^{j-1}\right)-n D \\
& =\sum_{j=1}^{n} H\left(X_{j} \mid F_{A^{c}}, X^{j-1}\right)+\sum_{i \in A} \sum_{j=1}^{n} I\left(Y_{i, j} ; F_{i} \mid X^{n}, Y_{i}^{j-1}\right)-n D \\
& =\sum_{j=1}^{n} H\left(X_{j} \mid F_{A^{c}}, X^{j-1}\right)+\sum_{i \in A} \sum_{j=1}^{n} I\left(Y_{i, j} ; U_{i, j} \mid X_{j}, Q_{j}\right)-n D \\
& \geq \sum_{j=1}^{n} H\left(X_{j} \mid U_{A^{c}, j}, Q_{j}\right)+\sum_{i \in A} \sum_{j=1}^{n} I\left(Y_{i, j} ; U_{i, j} \mid X_{j}, Q_{j}\right)-n D .
\end{aligned}
$$

The nontrivial steps above can be justified as follows:

- 2 follows since $F_{A}$ is a function of $Y_{A}^{n}$. 
- (3) follows since $F_{i}$ is a function of $Y_{i}^{n}$ and $F_{1} \leftrightarrow X^{n} \leftrightarrow F_{2}$ form a Markov chain (since $Y_{1}^{n} \leftrightarrow X^{n} \leftrightarrow Y_{2}^{n}$ form a Markov chain).

- (4) follows since $n D \geq H\left(X^{n} \mid F_{1}, F_{2}\right)$ by Lemma 1 .

- (5) follows from the Markov chain $Y_{i, j} \leftrightarrow X^{n} \leftrightarrow Y_{i}^{j-1}$, which follows from the i.i.d. nature of the source sequences.

- 66 simply follows from the fact that conditioning reduces entropy.

Therefore, dividing both sides by $n$, we have:

$$
\sum_{i \in A} R_{i} \geq \frac{1}{n} \sum_{j=1}^{n} H\left(X_{j} \mid U_{A^{c}, j}, Q_{j}\right)+\sum_{i \in A} \frac{1}{n} \sum_{j=1}^{n} I\left(Y_{i, j} ; U_{i, j} \mid X_{j}, Q_{j}\right)-D .
$$

Also, using Lemma 1 and the fact that conditioning reduces entropy:

$$
D \geq \frac{1}{n} H\left(X^{n} \mid F_{1}, F_{2}\right) \geq \frac{1}{n} \sum_{j=1}^{n} H\left(X_{j} \mid U_{1, j}, U_{2, j}, Q_{j}\right) .
$$

Observe that $Q_{j}$ is independent of $\left(X_{j}, Y_{1, j}, Y_{2, j}\right)$ and, conditioned on $Q_{j}$, we have the long Markov chain $U_{1, j} \leftrightarrow Y_{1, j} \leftrightarrow X_{j} \leftrightarrow Y_{2, j} \leftrightarrow U_{2, j}$. Finally, by a standard time-sharing argument, we conclude by saying that if $\left(R_{1}, R_{2}, D\right)$ is strict-sense achievable for the CEO problem, then

$$
\begin{aligned}
R_{1} & \geq I\left(Y_{1} ; U_{1} \mid X, Q\right)+H\left(X \mid U_{2}, Q\right)-D \\
R_{2} & \geq I\left(Y_{2} ; U_{2} \mid X, Q\right)+H\left(X \mid U_{1}, Q\right)-D \\
R_{1}+R_{2} & \geq I\left(U_{1} ; Y_{1} \mid X, Q\right)+I\left(U_{2} ; Y_{2} \mid X, Q\right)+H(X)-D \\
D & \geq H\left(X \mid U_{1}, U_{2}, Q\right) .
\end{aligned}
$$

for some joint distribution $p(q) p\left(x, y_{1}, y_{2}\right) p\left(u_{1} \mid y_{1}, q\right) p\left(u_{2} \mid y_{2}, q\right)$.

Theorem 3. $\mathcal{R D}_{C E O}^{o}=\mathcal{R} \mathcal{D}_{C E O}^{i}=\overline{\mathcal{R D}}_{C E O}^{\star}$.

Proof. We first remark that the cardinality bounds on the alphabets in the definition of $\mathcal{R} \mathcal{D}_{C E O}^{i}$ can be imposed without any loss of generality. This is a consequence of [17, Lemma 2.2] and is discussed in detail in Appendix A.

Therefore, it will suffice to show $\mathcal{R D}_{C E O}^{o} \subseteq \mathcal{R D}_{C E O}^{i}$ without considering the cardinality bounds. To this end, fix $p(q), p\left(u_{1} \mid y_{1}, q\right)$, and $p\left(u_{2} \mid y_{2}, q\right)$ and 
consider the extreme point ${ }^{1}$ of polytope defined by the inequalities (1):

$$
\begin{aligned}
& P_{1}=\left(0,0, I\left(Y_{1} ; U_{1} \mid X, Q\right)+I\left(Y_{2} ; U_{2} \mid X, Q\right)+H(X)\right) \\
& P_{2}=\left(I\left(Y_{1} ; U_{1} \mid Q\right), 0, I\left(U_{2} ; Y_{2} \mid X, Q\right)+H\left(X \mid U_{1}, Q\right)\right) \\
& P_{3}=\left(0, I\left(Y_{2} ; U_{2} \mid Q\right), I\left(U_{1} ; Y_{1} \mid X, Q\right)+H\left(X \mid U_{2}, Q\right)\right) \\
& P_{4}=\left(I\left(Y_{1} ; U_{1} \mid Q\right), I\left(Y_{2} ; U_{2} \mid U_{1}, Q\right), H\left(X \mid U_{1}, U_{2}, Q\right)\right) \\
& P_{5}=\left(I\left(Y_{1} ; U_{1} \mid U_{2}, Q\right), I\left(Y_{2} ; U_{2} \mid Q\right), H\left(X \mid U_{1}, U_{2}, Q\right)\right),
\end{aligned}
$$

where the point $P_{j}$ is a triple $\left(R_{1}^{(j)}, R_{2}^{(j)}, D^{(j)}\right)$. We say a point $\left(R_{1}^{(j)}, R_{2}^{(j)}, D^{(j)}\right)$ is dominated by a point in $\mathcal{R D}_{C E O}^{i}$ if there exists some $\left(R_{1}, R_{2}, D\right) \in \mathcal{R} \mathcal{D}_{C E O}^{i}$ for which $R_{1} \leq R_{1}^{(j)}, R_{2} \leq R_{2}^{(j)}$, and $D \leq D^{(j)}$. Observe that each of the extreme points $P_{1}, \ldots, P_{5}$ is dominated by a point in $\mathcal{R} \mathcal{D}_{C E O}^{i}$ :

- First, observe that $P_{4}$ and $P_{5}$ are both in $\mathcal{R} \mathcal{D}_{C E O}^{i}$, so these points are not problematic.

- Next, observe that the point $(0,0, H(X))$ is in $\mathcal{R D}_{C E O}^{i}$, which can be seen by setting all auxiliary random variables to be constant. This point dominates $P_{1}$.

- By using auxiliary random variables $\left(\hat{U}_{1}, \hat{U}_{2}, Q\right)=\left(U_{1}, \emptyset, Q\right)$, the point $\left(I\left(Y_{1} ; U_{1} \mid Q\right), 0, H\left(X \mid U_{1}, Q\right)\right)$ is in $\mathcal{R D}_{C E O}^{i}$, and dominates the point $P_{2}$. By a symmetric argument, the point $P_{3}$ is also dominated by a point in $\mathcal{R} \mathcal{D}_{C E O}^{i}$.

Since $\mathcal{R} \mathcal{D}_{C E O}^{o}$ is the convex hull of all such extreme points (i.e., the convex hull of the union of extreme points over all appropriate joint distributions), the theorem is proved.

Remark 1. Theorem 3 can be extended to the general case of m-encoders. Details are provided in Appendix $B$.

\subsection{A stronger converse result for the CEO problem}

As defined, our reproduction sequence $\hat{X}^{n}$ is restricted to be a product distribution on $\mathcal{X}^{n}$. However, for a blocklength $n$ code, we can allow $\hat{X}^{n}$ to be any probability distribution on $\mathcal{X}^{n}$ and the converse result still holds. In this case, we define the sequence distortion as follows:

$$
d\left(x^{n}, \hat{x}^{n}\right)=\frac{1}{n} \log \left(\frac{1}{\hat{x}^{n}\left(x^{n}\right)}\right),
$$

\footnotetext{
${ }^{1}$ For two encoders, it is easy enough to enumerate the extreme points by inspection. However, this can be formalized by a submodularity argument, which is given in Appendix B
} 
which is compatible with the original definition when $\hat{X}^{n}$ is a product distribution. The reader can verify that the result of Lemma 1 is still true for this more general distortion alphabet by setting $s\left(x^{n} \mid z\right)=\hat{x}^{n}\left(x^{n}\right)$ in the corresponding proof. Since Lemma 1 is the key tool in the CEO converse result, this implies that the converse holds even if $\hat{X}^{n}$ is allowed to be any probability distribution on $\mathcal{X}^{n}$ (rather than being restricted to the set of product distributions).

When this stronger converse result is taken together with the achievability result, we observe that restricting $\hat{X}^{n}$ to be a product distribution is in fact optimal and can achieve all points in $\overline{\mathcal{R D}}_{C E O}^{\star}$.

\subsection{An Example: Distributed compression of a posterior distribution}

Suppose two sensors observe sequences $Y_{1}^{n}$ and $Y_{2}^{n}$ respectively, which are conditionally independent given a hidden sequence $X^{n}$. The sensors communicate with a fusion center through rate-limited links of capacity $R_{1}$ and $R_{2}$ respectively. Given sequences $\left(Y_{1}^{n}, Y_{2}^{n}\right)$ are observed, the sequence $X^{n}$ cannot be determined in general, so the fusion center would like to estimate the posterior distribution $p\left(x^{n} \mid Y_{1}^{n}, Y_{2}^{n}\right)$. Since the communication links are rate-limited, the fusion center cannot necessarily compute $p\left(x^{n} \mid Y_{1}^{n}, Y_{2}^{n}\right)$ exactly. In this case, the fusion center would like to generate an estimate $\hat{p}\left(x^{n} \mid g_{1}^{(n)}\left(Y_{1}^{n}\right), g_{2}^{(n)}\left(Y_{2}^{n}\right)\right)$ that should approximate $p\left(x^{n} \mid Y_{1}^{n}, Y_{2}^{n}\right)$ in the sense that, on average:

$$
D\left(p\left(x^{n} \mid y_{1}^{n}, y_{2}^{n}\right) \| \hat{p}\left(x^{n} \mid g_{1}^{(n)}\left(y_{1}^{n}\right), g_{2}^{(n)}\left(y_{2}^{n}\right)\right)\right) \leq n \varepsilon
$$

where, consistent with standard notation (e.g. [18]), we write $D\left(p\left(x^{n} \mid y_{1}^{n}, y_{2}^{n}\right) \| \hat{p}\left(x^{n} \mid g_{1}^{(n)}\left(y_{1}^{n}\right), g_{2}^{(n)}\left(y_{2}^{n}\right)\right)\right)$ as shorthand for

$$
\sum_{x^{n}, y_{1}^{n}, y_{2}^{n}} p\left(x^{n}, y_{1}^{n}, y_{2}^{n}\right) \log \frac{p\left(x^{n} \mid y_{1}^{n}, y_{2}^{n}\right)}{\hat{p}\left(x^{n} \mid g_{1}^{(n)}\left(y_{1}^{n}\right), g_{2}^{(n)}\left(y_{2}^{n}\right)\right)} .
$$

The relevant question here is the following. What is the minimum distortion $\varepsilon$ that is attainable given $R_{1}$ and $R_{2}$ ?

Considering the CEO problem for this setup, we have:

$$
\begin{aligned}
\mathbb{E} d\left(\hat{X}^{n}, X^{n}\right) & =\frac{1}{n} \sum_{\left(x^{n}, y_{1}^{n}, y_{2}^{n}\right)} p\left(x^{n}, y_{1}^{n}, y_{2}^{n}\right) \log \left(\frac{1}{\hat{x}^{n}\left(x^{n}\right)}\right) \\
& =\frac{1}{n} D\left(p\left(x^{n} \mid y_{1}^{n}, y_{2}^{n}\right) \| \hat{x}^{n}\left(x^{n}\right)\right)+\frac{1}{n} H\left(X^{n} \mid Y_{1}^{n}, Y_{2}^{n}\right) .
\end{aligned}
$$

Identifying $\hat{p}\left(x^{n} \mid g_{1}^{(n)}\left(Y_{1}^{n}\right), g_{2}^{(n)}\left(Y_{2}^{n}\right)\right) \leftarrow \hat{X}^{n}\left(x^{n}\right)$, we have:

$$
D\left(p\left(x^{n} \mid y_{1}^{n}, y_{2}^{n}\right) \| \hat{p}\left(x^{n} \mid g_{1}^{(n)}\left(y_{1}^{n}\right), g_{2}^{(n)}\left(y_{2}^{n}\right)\right)\right)=n \mathbb{E} d\left(\hat{X}^{n}, X^{n}\right)-n H\left(X \mid Y_{1}, Y_{2}\right) .
$$




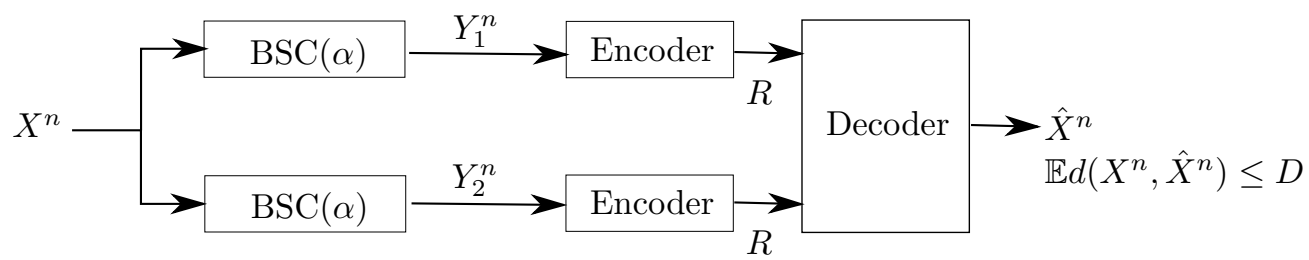

Figure 2: An example CEO problem where $X \sim \operatorname{Bernoulli}\left(\frac{1}{2}\right), \operatorname{Pr}\left(Y_{i}=X\right)=$ $(1-\alpha)$, and both encoders are subject to the same rate constraint.

Thus, finding the minimum possible distortion reduces to an optimization problem over $\overline{\mathcal{R D}}_{C E O}^{\star}$. In particular, the minimum attainable distortion $\varepsilon^{*}$ is given by

$$
\varepsilon^{*}=\inf \left\{D:\left(R_{1}, R_{2}, D\right) \in \overline{\mathcal{R D}}_{C E O}^{\star}\right\}-H\left(X \mid Y_{1}, Y_{2}\right)
$$

Moreover, the minimum distortion is obtained by estimating each $x_{j}$ separately. In other words, there exists an optimal (essentially, for large $n$ ) estimate $\hat{p}^{*}\left(x^{n} \mid \cdot, \cdot\right)$ (which is itself a function of optimal encoding functions $g_{1}^{*(n)}(\cdot)$ and $\left.g_{2}^{*(n)}(\cdot)\right)$ that can be expressed as a product distribution

$$
\hat{p}^{*}\left(x^{n} \mid \cdot, \cdot\right)=\prod_{j=1}^{n} \hat{p}_{j}^{*}\left(x_{j} \mid g_{1}^{*(n)}(\cdot), g_{2}^{*(n)}(\cdot)\right)
$$

For this choice of $\hat{p}^{*}\left(x^{n} \mid \cdot, \cdot\right)$, we have the following relationship:

$$
\frac{1}{n} \sum_{j=1}^{n} D\left(p\left(x_{j} \mid y_{1, j}, y_{2, j}\right) \| \hat{p}_{j}^{*}\left(x_{j} \mid g_{1}^{*(n)}\left(y_{1}^{n}\right), g_{2}^{*(n)}\left(y_{2}^{n}\right)\right)\right)=\varepsilon^{*}
$$

In light of this fact, one can apply Markov's inequality to obtain the following estimate on peak component-wise distortion:

$$
\#\left\{j \mid D\left(p\left(x_{j} \mid y_{1, j}, y_{2, j}\right) \| \hat{p}_{j}^{*}\left(x_{j} \mid g_{1}^{*(n)}\left(y_{1}^{n}\right), g_{2}^{*(n)}\left(y_{2}^{n}\right)\right)\right) \geq \zeta\right\} \leq n \frac{\varepsilon^{*}}{\zeta}
$$

where \#(·) is the counting measure.

To make this example more concrete, consider the scenario depicted in Figure 2. where $X \sim \operatorname{Bernoulli}\left(\frac{1}{2}\right)$ and $Y_{i}$ is the result of passing $X$ through a binary symmetric channel with crossover probability $\alpha$ for $i=1,2$. To simplify things, we constrain the rates of each encoder to be at most $R$ bits per channel use.

By performing a brute-force search over a fine mesh of conditional distributions $\left\{p\left(u_{i} \mid y_{i}\right)\right\}_{i=1}^{2}$, we numerically approximate the set of $(R, D)$ pairs such that $(R, R, D)$ is in the achievable region $\overline{\mathcal{R D}}_{C E O}^{\star}$ corresponding to the network in Figure 2. The lower convex envelope of these $(R, D)$ pairs is plotted in Figure 3 


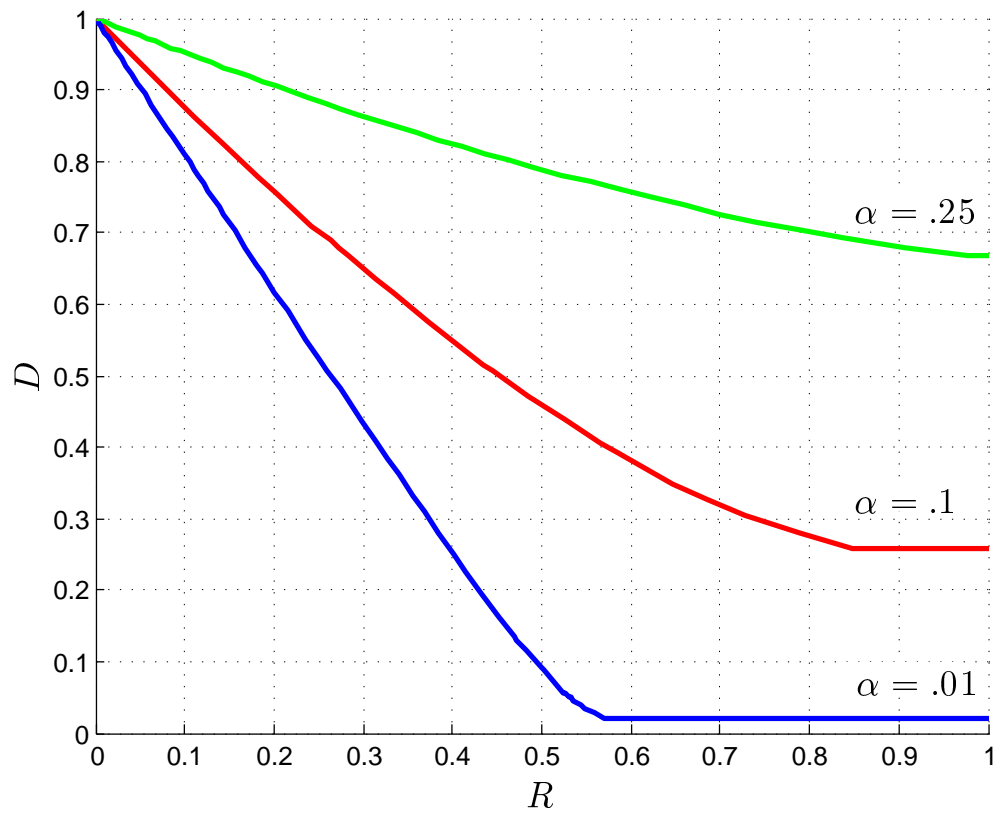

Figure 3: The distortion-rate function of the network in Figure 2 computed for $\alpha \in\{0.01,0.1,0.25\}$.

for $\alpha \in\{0.01,0.1,0.25\}$. Continuing our example above for this concrete choice of source parameters, we compute the minimum achievable Kullback-Leibler distance $\varepsilon^{*}$ according to $(7)$. The result is given in Figure 4

These numerical results are intuitively satisfying in the sense that, if $Y_{1}, Y_{2}$ are high-quality estimates of $X$ (e.g., $\alpha=0.01$ ), then a small increase in the allowable rate $R$ results in a large relative improvement of $\hat{p}(x \mid \cdot, \cdot)$, the decoder's estimate of $p\left(x \mid Y_{1}, Y_{2}\right)$. On the other hand, if $Y_{1}, Y_{2}$ are poor-quality estimates of $X$ (e.g., $\alpha=0.25$ ), then we require a large increase in the allowable rate $R$ in order to obtain an appreciable improvement of $\hat{p}(x \mid \cdot, \cdot)$.

One field where this example is directly applicable is machine learning. In this case, $X_{j}$ could represent the class of object $j$, and $Y_{1, j}, Y_{2, j}$ are observable attributes. In machine learning, one typically estimates the probability that an object belongs to a particular class given a set of observable attributes. For this type of estimation problem, relative entropy is a natural penalty criterion.

Another application is to horse-racing with conditionally independent, ratelimited side informations. In this case, the doubling rate of the gambler's wealth can be expressed in terms of the logarithmic loss distortion measure. This example is consistent with the original interpretation of the CEO problem, where the CEO makes consecutive business decisions (investments) having outcomes $X^{n}$, with the objective of maximizing the wealth of the company. We omit the details. 


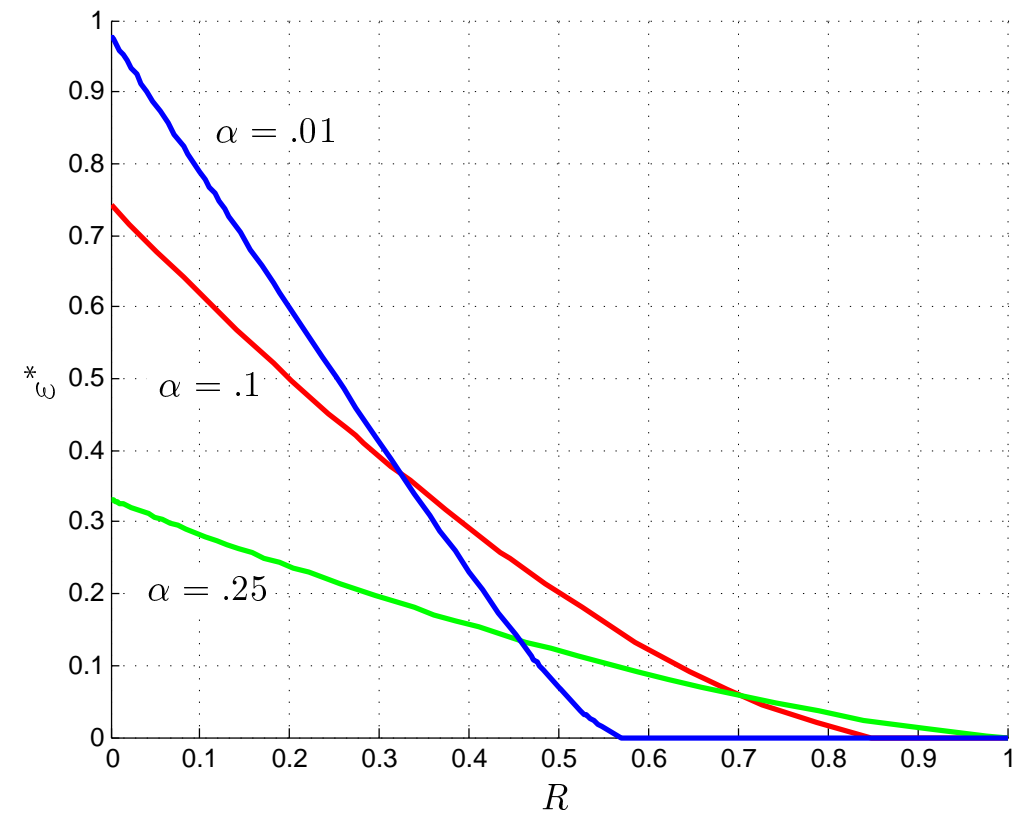

Figure 4: The minimum achievable Kullback-Leibler distance computed according to 77 , i.e., the curves here are those of Figure 3, lowered by the constant $H\left(X \mid Y_{1}, Y_{2}\right)$.

\subsection{An Example: Joint estimation of the encoder obser- vations}

Suppose one wishes to estimate the encoder observations $\left(Y_{1}, Y_{2}\right)$. In this case, the rate region simplifies considerably. In particular, if we tolerate a distortion $D$ in our estimate of the pair $\left(Y_{1}, Y_{2}\right)$, then the achievable rate region is the same as the Slepian-Wolf rate region with each rate constraint relaxed by $D$ bits. Formally:

Theorem 4. If $X=\left(Y_{1}, Y_{2}\right)$, then $\overline{\mathcal{R D}}_{C E O}^{\star}$ consists of all vectors $\left(R_{1}, R_{2}, D\right)$ satisfying

$$
\begin{aligned}
R_{1} & \geq H\left(Y_{1} \mid Y_{2}\right)-D \\
R_{2} & \geq H\left(Y_{2} \mid Y_{1}\right)-D \\
R_{1}+R_{2} & \geq H\left(Y_{1}, Y_{2}\right)-D \\
D & \geq 0 .
\end{aligned}
$$

Proof. First, note that Theorem 3 implies that $\overline{\mathcal{R D}}_{C E O}^{\star}$ is equivalent to the the union of $\left(R_{1}, R_{2}, D\right)$ triples satisfying (1) taken over all joint distributions $p(q) p\left(x, y_{1}, y_{2}\right) p\left(u_{1} \mid y_{1}, q\right) p\left(u_{2} \mid y_{2}, q\right)$. Now, since $X=\left(Y_{1}, Y_{2}\right)$, each of the in- 
equalities (1) can be lower bounded as follows:

$$
\begin{aligned}
R_{1} & \geq I\left(Y_{1} ; U_{1} \mid Y_{1}, Y_{2}, Q\right)+H\left(Y_{1}, Y_{2} \mid U_{2}, Q\right)-D \\
& =H\left(Y_{2} \mid U_{2}, Q\right)+H\left(Y_{1} \mid Y_{2}\right)-D \\
& \geq H\left(Y_{1} \mid Y_{2}\right)-D \\
R_{2} & \geq I\left(Y_{2} ; U_{2} \mid Y_{1}, Y_{2}, Q\right)+H\left(Y_{1}, Y_{2} \mid U_{1}, Q\right)-D \\
& =H\left(Y_{1} \mid U_{1}, Q\right)+H\left(Y_{2} \mid Y_{1}\right)-D \\
& \geq H\left(Y_{2} \mid Y_{1}\right)-D \\
R_{1}+R_{2} & \geq I\left(U_{1} ; Y_{1} \mid Y_{1}, Y_{2}, Q\right)+I\left(U_{2} ; Y_{2} \mid Y_{1}, Y_{2}, Q\right)+H\left(Y_{1}, Y_{2}\right)-D \\
& =H\left(Y_{1}, Y_{2}\right)-D \\
D & \geq H\left(Y_{1}, Y_{2} \mid U_{1}, U_{2}, Q\right) \\
& \geq 0 .
\end{aligned}
$$

Finally, observe that by setting $U_{i}=Y_{i}$ for $i=1,2$, we can achieve any point in this relaxed region (again, a consequence of Theorem 3 ).

We remark that this result was first proved in [10 by Courtade and Wesel using a different method.

\section{Multiterminal Source Coding}

With Theorem 3 in hand, we are now in a position to characterize the achievable rate distortion region $\overline{\mathcal{R D}}^{\star}$ for the multiterminal source coding problem under logarithmic loss. As before, we prove an inner bound first.

\subsection{Inner Bound}

Definition 5. Let $\left(R_{1}, R_{2}, D_{1}, D_{2}\right) \in \mathcal{R D}^{i}$ if and only if there exists a joint distribution of the form

$$
p\left(y_{1}, y_{2}\right) p\left(u_{1} \mid y_{1}, q\right) p\left(u_{2} \mid y_{2}, q\right) p(q)
$$

where $\left|\mathcal{U}_{1}\right| \leq\left|\mathcal{Y}_{1}\right|,\left|\mathcal{U}_{2}\right| \leq\left|\mathcal{Y}_{2}\right|$, and $|\mathcal{Q}| \leq 5$, which satisfies

$$
\begin{aligned}
R_{1} & \geq I\left(Y_{1} ; U_{1} \mid U_{2}, Q\right) \\
R_{2} & \geq I\left(Y_{2} ; U_{2} \mid U_{1}, Q\right) \\
R_{1}+R_{2} & \geq I\left(U_{1}, U_{2} ; Y_{1}, Y_{2} \mid Q\right) \\
D_{1} & \geq H\left(Y_{1} \mid U_{1}, U_{2}, Q\right) \\
D_{2} & \geq H\left(Y_{2} \mid U_{1}, U_{2}, Q\right) .
\end{aligned}
$$

Theorem 5. $\mathcal{R D}^{i} \subseteq \overline{\mathcal{R D}}^{\star}$. That is, all rate distortion vectors in $\mathcal{R D}^{i}$ are achievable.

Again, we require an appropriate version of the Berger-Tung inner bound: 
Proposition 2 (Berger-Tung Inner Bound 14,15]). The rate distortion vector $\left(R_{1}, R_{2}, D_{1}, D_{2}\right)$ is achievable if

$$
\begin{aligned}
R_{1} & \geq I\left(U_{1} ; Y_{1} \mid U_{2}, Q\right) \\
R_{2} & \geq I\left(U_{2} ; Y_{2} \mid U_{1}, Q\right) \\
R_{1}+R_{2} & \geq I\left(U_{1}, U_{2} ; Y_{1}, Y_{2} \mid Q\right) \\
D_{1} & \geq \mathbb{E}\left[d\left(Y_{1}, f_{1}\left(U_{1}, U_{2}, Q\right)\right]\right. \\
D_{2} & \geq \mathbb{E}\left[d\left(Y_{2}, f_{2}\left(U_{1}, U_{2}, Q\right)\right] .\right.
\end{aligned}
$$

for a joint distribution

$$
p\left(y_{1}, y_{2}\right) p\left(u_{1} \mid y_{1}, q\right) p\left(u_{2} \mid y_{2}, q\right) p(q)
$$

and reproduction functions

$$
f_{i}: \mathcal{U}_{1} \times \mathcal{U}_{2} \times \mathcal{Q} \rightarrow \hat{\mathcal{Y}}_{i}, \text { for } i=1,2 .
$$

Proof of Theorem 5. To prove the theorem, we simply apply Proposition 2 with the reproduction functions $f_{i}\left(U_{1}, U_{2}, Q\right):=\operatorname{Pr}\left[Y_{i}=y_{i} \mid U_{1}, U_{2}, Q\right]$.

Hence, we again see that our inner bound $\mathcal{R D}^{i} \subseteq \overline{\mathcal{R D}}^{\star}$ is nothing more than the Berger-Tung inner bound specialized to the setting when distortion is measured under logarithmic loss.

\subsection{A Matching Outer Bound}

The main result of this paper is the following theorem.

Theorem 6. $\mathcal{R D}^{i}=\overline{\mathcal{R D}}^{\star}$.

Proof. As before, we note that the cardinality bounds on the alphabets in the definition of $\mathcal{R} \mathcal{D}^{i}$ can be imposed without any loss of generality. This is discussed in detail in Appendix A.

Assume $\left(R_{1}, R_{2}, D_{1}, D_{2}\right)$ is strict-sense achievable. Observe that proving that $\left(R_{1}, R_{2}, D_{1}, D_{2}\right) \in \mathcal{R} \mathcal{D}^{i}$ will prove the theorem, since $\mathcal{R} \mathcal{D}^{i} \subseteq \overline{\mathcal{R D}}^{\star}$ and $\overline{\mathcal{R D}}^{\star}$ is closed by definition.

For convenience, define $\mathcal{P}\left(R_{1}, R_{2}\right)$ to be the set of joint distributions of the form

$$
p\left(y_{1}, y_{2}\right) p\left(u_{1} \mid y_{1}, q\right) p\left(u_{2} \mid y_{2}, q\right) p(q)
$$

with $\left|\mathcal{U}_{1}\right| \leq\left|\mathcal{Y}_{1}\right|,\left|\mathcal{U}_{2}\right| \leq\left|\mathcal{Y}_{2}\right|$, and $|\mathcal{Q}| \leq 4$ satisfying

$$
\begin{aligned}
R_{1} & \geq I\left(U_{1} ; Y_{1} \mid U_{2}, Q\right) \\
R_{2} & \geq I\left(U_{2} ; Y_{2} \mid U_{1}, Q\right) \\
R_{1}+ & R_{2} \geq I\left(U_{1}, U_{2} ; Y_{1}, Y_{2} \mid Q\right) .
\end{aligned}
$$


We remark that $\mathcal{P}\left(R_{1}, R_{2}\right)$ is compact. We also note that it will suffice to show the existence of a joint distribution in $\mathcal{P}\left(R_{1}, R_{2}\right)$ satisfying $H\left(Y_{1} \mid U_{1}, U_{2}, Q\right) \leq$ $D_{1}$ and $H\left(Y_{2} \mid U_{1}, U_{2}, Q\right) \leq D_{2}$ to prove that $\left(R_{1}, R_{2}, D_{1}, D_{2}\right) \in \mathcal{R} \mathcal{D}^{i}$.

With foresight, consider random variable $X$ defined as follows

$$
X= \begin{cases}\left(Y_{1}, 1\right) & \text { with probability } t \\ \left(Y_{2}, 2\right) & \text { with probability } 1-t .\end{cases}
$$

In other words, $X=\left(Y_{B}, B\right)$, where $B$ is a Bernoulli random variable independent of $Y_{1}, Y_{2}$. Observe that $Y_{1} \leftrightarrow X \leftrightarrow Y_{2}$ form a Markov chain, and thus, we are able to apply Theorem 3 .

Since $\left(R_{1}, R_{2}, D_{1}, D_{2}\right)$ is strict-sense achievable, the decoder can construct reproductions $\hat{Y}_{1}^{n}, \hat{Y}_{2}^{n}$ satisfying

$$
\frac{1}{n} \sum_{j=1}^{n} \mathbb{E} d\left(Y_{i, j}, \hat{Y}_{i, j}\right) \leq D_{i} \text { for } i=1,2 .
$$

Fix the encoding operations and set $\hat{X}_{j}\left(\left(y_{1}, 1\right)\right)=t \hat{Y}_{1, j}\left(y_{1}\right)$ and $\hat{X}_{j}\left(\left(y_{2}, 2\right)\right)=$ $(1-t) \hat{Y}_{2, j}\left(y_{2}\right)$. Then for the CEO problem defined by $\left(X, Y_{1}, Y_{2}\right)$ :

$$
\begin{aligned}
& \frac{1}{n} \sum_{j=1}^{n} \mathbb{E} d\left(X_{j}, \hat{X}_{j}\right) \\
& =\frac{t}{n} \sum_{j=1}^{n} \mathbb{E} \log \left(\frac{1}{t \hat{Y}_{1, j}\left(Y_{1, j}\right)}\right)+\frac{1-t}{n} \sum_{j=1}^{n} \mathbb{E} \log \left(\frac{1}{(1-t) \hat{Y}_{2, j}\left(Y_{2, j}\right)}\right) \\
& =h_{2}(t)+\frac{t}{n} \sum_{j=1}^{n} \mathbb{E} d\left(Y_{1, j}, \hat{Y}_{1, j}\right)+\frac{1-t}{n} \sum_{j=1}^{n} \mathbb{E} d\left(Y_{2, j}, \hat{Y}_{2, j}\right) \\
& \leq h_{2}(t)+t D_{1}+(1-t) D_{2}
\end{aligned}
$$

where $h_{2}(t)$ is the binary entropy function. Hence, for this CEO problem, distortion $h_{2}(t)+t D_{1}+(1-t) D_{2}$ is achievable and Theorem 3 yields a joint distribution ${ }^{2} P_{t} \in \mathcal{P}\left(R_{1}, R_{2}\right)$ satisfying

$$
\begin{aligned}
h_{2}(t)+t D_{1}+(1-t) D_{2} \geq & H\left(X \mid U_{1}^{(t)}, U_{2}^{(t)}, Q^{(t)}\right) \\
= & h_{2}(t)+t H\left(Y_{1} \mid U_{1}^{(t)}, U_{2}^{(t)}, Q^{(t)}\right) \\
& +(1-t) H\left(Y_{2} \mid U_{1}^{(t)}, U_{2}^{(t)}, Q^{(t)}\right),
\end{aligned}
$$

where the second equality follows by by definition of $X$ in (8). For convenience, define $H_{1}\left(P_{t}\right) \triangleq H\left(Y_{1} \mid U_{1}^{(t)}, U_{2}^{(t)}, Q^{(t)}\right)$ and $H_{2}\left(P_{t}\right) \triangleq H\left(Y_{2} \mid U_{1}^{(t)}, U_{2}^{(t)}, Q^{(t)}\right)$. Note the following two facts:

1. By continuity of entropy, the functions $H_{1}(\cdot)$ and $H_{2}(\cdot)$ are continuous on the compact domain $\mathcal{P}\left(R_{1}, R_{2}\right)$.

\footnotetext{
${ }^{2}$ Henceforth, we use the superscript $(t)$ to explicitly denote the dependence of the auxiliary random variables on the distribution parametrized by $t$.
} 
2. The above argument proves the existence of a function $\varphi:[0,1] \rightarrow$ $\mathcal{P}\left(R_{1}, R_{2}\right)$ which satisfies

$$
t H_{1}(\varphi(t))+(1-t) H_{2}(\varphi(t)) \leq t D_{1}+(1-t) D_{2} \text { for all } t \in[0,1] .
$$

These two facts satisfy the requirements of Lemma 7 (see Appendix $\mathrm{D}$ ), and hence there exists $P_{t_{1}} \in \mathcal{P}\left(R_{1}, R_{2}\right), P_{t_{2}} \in \mathcal{P}\left(R_{1}, R_{2}\right)$, and $\theta \in[0,1]$ for which

$$
\begin{aligned}
& \theta H_{1}\left(P_{t_{1}}\right)+(1-\theta) H_{1}\left(P_{t_{2}}\right) \leq D_{1} \\
& \theta H_{2}\left(P_{t_{1}}\right)+(1-\theta) H_{2}\left(P_{t_{2}}\right) \leq D_{2} .
\end{aligned}
$$

Timesharing ${ }^{3}$ between distributions $P_{t_{1}}$ and $P_{t_{2}}$ with probabilities $\theta$ and (1$\theta)$, respectively, yields a distribution $P^{*} \in \mathcal{P}\left(R_{1}, R_{2}\right)$ which satisfies $H_{1}\left(P^{*}\right) \leq$ $D_{1}$ and $H_{2}\left(P^{*}\right) \leq D_{2}$. This proves the theorem.

\subsection{A stronger converse}

For the CEO problem, we are able to obtain a stronger converse result as discussed in Section 3.3. We can obtain a similar result for the multiterminal source coding problem. Indeed, the converse result we just proved continues to hold even when $\hat{Y}_{i}^{n}$ is allowed to be any probability measure on $\mathcal{Y}_{i}^{n}$, rather than a product distribution. The proof of this fact is somewhat involved and can be found in Appendix E.

We note that the proof of this strengthened converse result (i.e., Theorem 13 in Appendix Ep offers a direct proof of the converse of Theorem 6, and as such we do not require a CEO result (Theorem 3) or a "black box" tuning argument (Lemma 7). At the heart of this alternative proof lies the Csiszár sum identity (and a careful choice of auxiliary random variables) which provides a coupling between the attainable distortions for each source. In the original proof of Theorem 6, this coupling is accomplished by the tuning argument through Lemma 7.

Interestingly, the two proofs are similar in spirit, with the key differences being the use of the Csiszár sum identity versus the tuning argument. Intuitively, the original tuning argument allows a "clumsier" choice of auxiliary random variables which leads to a more elegant and transparent proof, but appears incapable of establishing the strengthened converse. On the other hand, applying the Csiszár sum identity requires a very careful choice of auxiliary random variables which, in turn, affords a finer degree of control over various quantities.

\subsection{An Example: The Daily Double}

The Daily Double is a single bet that links together wagers on the winners of two consecutive horse races. Winning the Daily Double is dependent on both wagers winning together. In general, the outcomes of two consecutive races can

\footnotetext{
${ }^{3}$ The timesharing scheme can be embedded in the timesharing variable $Q$, increasing the cardinality of $\mathcal{Q}$ by a factor of two.
} 
be correlated (e.g. due to track conditions), so a gambler can potentially use this information to maximize his expected winnings. Let $\mathcal{Y}_{1}$ and $\mathcal{Y}_{2}$ be the set of horses running in the first and second races respectively. If horses $y_{1}$ and $y_{2}$ win their respective races, then the payoff is $o\left(y_{1}, y_{2}\right)$ dollars for each dollar invested in outcome $\left(Y_{1}, Y_{2}\right)=\left(y_{1}, y_{2}\right)$.

There are two betting strategies one can follow:

1. The gambler can wager a fraction $b_{1}\left(y_{1}\right)$ of his wealth on horse $y_{1}$ winning the first race and parlay his winnings by betting a fraction $b_{2}\left(y_{2}\right)$ of his wealth on horse $y_{2}$ winning the second race. In this case, the gambler's wealth relative is $b_{1}\left(Y_{1}\right) b_{2}\left(Y_{2}\right) o\left(Y_{1}, Y_{2}\right)$ upon learning the outcome of the Daily Double. We refer to this betting strategy as the product-wager.

2. The gambler can wager a fraction $b\left(y_{1}, y_{2}\right)$ of his wealth on horses $\left(y_{1}, y_{2}\right)$ winning the first and second races, respectively. In this case, the gambler's wealth relative is $b\left(Y_{1}, Y_{2}\right) o\left(Y_{1}, Y_{2}\right)$ upon learning the outcome of the Daily Double. We refer to this betting strategy as the joint-wager.

Clearly the joint-wager includes the product-wager as a special case. However, the product-wager requires less effort to place, so the question is: how do the two betting strategies compare?

To make things interesting, suppose the gamblers have access to rate-limited information about the first and second race outcomes at rates $R_{1}, R_{2}$ respectively. Further, assume that $R_{1} \leq H\left(Y_{1}\right), R_{2} \leq H\left(Y_{2}\right)$, and $R_{1}+R_{2} \leq$ $H\left(Y_{1}, Y_{2}\right)$. For $\left(R_{1}, R_{2}\right)$ and $p\left(y_{1}, y_{2}\right)$ given, let $\mathcal{P}\left(R_{1}, R_{2}\right)$ denote the set of joint pmf's of the form

$$
p\left(q, y_{1}, y_{2}, u_{1}, u_{2}\right)=p(q) p\left(y_{1}, y_{2}\right) p\left(u_{1} \mid y_{1}, q\right) p\left(u_{1} \mid y_{1}, q\right)
$$

which satisfy

$$
\begin{aligned}
R_{1} \geq I\left(Y_{1} ; U_{1} \mid U_{2}, Q\right) \\
R_{2} \geq I\left(Y_{2} ; U_{2} \mid U_{1}, Q\right) \\
R_{1}+R_{2} \geq I\left(Y_{1}, Y_{2} ; U_{1}, U_{2} \mid Q\right)
\end{aligned}
$$

for alphabets $\mathcal{U}_{1}, \mathcal{U}_{2}, \mathcal{Q}$ satisfying $\left|\mathcal{U}_{i}\right| \leq\left|\mathcal{Y}_{i}\right|$ and $|Q| \leq 5$.

Typically, the quality of a bet is measured by the associated doubling rate (cf. [18]). Theorem 6 implies that the optimal doubling rate for the productwager is given by:

$$
\begin{aligned}
W_{\mathrm{p}-\mathrm{w}}^{*}\left(p\left(y_{1}, y_{2}\right)\right) & =\sum_{y_{1}, y_{2}} p\left(y_{1}, y_{2}\right) \log b_{1}^{*}\left(y_{1}\right) b_{2}^{*}\left(y_{2}\right) o\left(y_{1}, y_{2}\right) \\
& =\mathbb{E} \log o\left(Y_{1}, Y_{2}\right)-\inf _{p \in \mathcal{P}\left(R_{1}, R_{2}\right)}\left\{H\left(Y_{1} \mid U_{1}, U_{2}, Q\right)+H\left(Y_{2} \mid U_{1}, U_{2}, Q\right)\right\}
\end{aligned}
$$

Likewise, Theorem 4 implies that the optimal doubling rate for the joint-wager 
is given by:

$$
\begin{aligned}
W_{\mathrm{j}-\mathrm{w}}^{*}\left(p\left(y_{1}, y_{2}\right)\right)= & \sum_{y_{1}, y_{2}} p\left(y_{1}, y_{2}\right) \log b^{*}\left(y_{1}, y_{2}\right) o\left(y_{1}, y_{2}\right) \\
= & \mathbb{E} \log o\left(Y_{1}, Y_{2}\right)+\min \left\{R_{1}-H\left(Y_{1} \mid Y_{2}\right), R_{2}-H\left(Y_{2} \mid Y_{1}\right),\right. \\
& \left.R_{1}+R_{2}-H\left(Y_{1}, Y_{2}\right)\right\} .
\end{aligned}
$$

It is important to note that we do not require the side informations to be the same for each type of wager, rather, the side informations are only provided at the same rates. Thus, the gambler placing the joint-wager receives side information at rates $\left(R_{1}, R_{2}\right)$ that maximizes his doubling rate, while the gambler placing the product-wager receives (potentially different) side information at rates $\left(R_{1}, R_{2}\right)$ that maximizes his doubling rate. However, as we will see shortly, for any rates $\left(R_{1}, R_{2}\right)$, there always exists rate-limited side information which simultaneously allows each type of gambler to attain their maximum doubling rate.

By combining the expressions for $W_{\mathrm{p}-\mathrm{w}}^{*}\left(p\left(y_{1}, y_{2}\right)\right)$ and $W_{\mathrm{j}-\mathrm{w}}^{*}\left(p\left(y_{1}, y_{2}\right)\right)$, we find that the difference in doubling rates is given by:

$$
\begin{aligned}
\Delta & \left(R_{1}, R_{2}\right)=W_{\mathrm{j}-\mathrm{w}}^{*}\left(p\left(y_{1}, y_{2}\right)\right)-W_{\mathrm{p}-\mathrm{w}}^{*}\left(p\left(y_{1}, y_{2}\right)\right) \\
= & \min \left\{R_{1}-H\left(Y_{1} \mid Y_{2}\right), R_{2}-H\left(Y_{2} \mid Y_{1}\right), R_{1}+R_{2}-H\left(Y_{1}, Y_{2}\right)\right\} \\
& +\inf _{p \in \mathcal{P}\left(R_{1}, R_{2}\right)}\left\{H\left(Y_{1} \mid U_{1}, U_{2}, Q\right)+H\left(Y_{2} \mid U_{1}, U_{2}, Q\right)\right\} \\
= & \inf _{p \in \mathcal{P}\left(R_{1}, R_{2}\right)} \min \left\{R_{1}-I\left(Y_{1} ; U_{1} \mid U_{2}, Q\right)+I\left(Y_{1} ; Y_{2}\right)-I\left(Y_{1} ; U_{2}, Q\right)+H\left(Y_{2} \mid U_{1}, U_{2}, Q\right)\right. \\
& R_{2}-I\left(Y_{2} ; U_{2} \mid U_{1}, Q\right)+I\left(Y_{2} ; Y_{1}\right)-I\left(Y_{2} ; U_{1}, Q\right)+H\left(Y_{1} \mid U_{1}, U_{2}, Q\right) \\
& \left.R_{1}+R_{2}-I\left(Y_{1}, Y_{2} ; U_{1}, U_{2} \mid Q\right)+I\left(Y_{1} ; Y_{2} \mid U_{1}, U_{2}, Q\right)\right\} \\
= & \inf _{p \in \mathcal{P}\left(R_{1}, R_{2}\right)} I\left(Y_{1} ; Y_{2} \mid U_{1}, U_{2}, Q\right) .
\end{aligned}
$$

The final equality $(10)$ follows since

- $R_{1} \geq I\left(Y_{1} ; U_{1} \mid U_{2}, Q\right)$ and $R_{2} \geq I\left(Y_{2} ; U_{2} \mid U_{1}, Q\right)$ for any $p \in \mathcal{P}\left(R_{1}, R_{2}\right)$.

- $I\left(Y_{2} ; Y_{1}\right) \geq I\left(Y_{2} ; U_{1}, Q\right)$ and $I\left(Y_{1} ; Y_{2}\right) \geq I\left(Y_{1} ; U_{2}, Q\right)$ for any $p \in$ $\mathcal{P}\left(R_{1}, R_{2}\right)$ by the data processing inequality.

- The infimum in (9) is attained by a $p \in \mathcal{P}\left(R_{1}, R_{2}\right)$ satisfying $R_{1}+R_{2}=$ $I\left(Y_{1}, Y_{2} ; U_{1}, U_{2} \mid Q\right)$. See Lemma 10 in Appendix $\mathrm{F}$ for details.

- By definition of conditional mutual information,

$$
H\left(Y_{i} \mid U_{1}, U_{2}, Q\right) \geq I\left(Y_{1} ; Y_{2} \mid U_{1}, U_{2}, Q\right)
$$

for $i=1,2$. 
Let $p^{*} \in \mathcal{P}\left(R_{1}, R_{2}\right)$ be the distribution that attains the infimum in (9) (such a $p^{*}$ always exists), then (10) yields

$$
\begin{aligned}
& W_{\mathrm{j}-\mathrm{W}}^{*}\left(p\left(y_{1}, y_{2}\right)\right)-W_{\mathrm{p}-\mathrm{W}}^{*}\left(p\left(y_{1}, y_{2}\right)\right) \\
& =\sum_{u_{1}, u_{2}, q} p^{*}\left(u_{1}, u_{2}, q\right) \sum_{y_{1}, y_{2}} p^{*}\left(y_{1}, y_{2} \mid u_{1}, u_{2}, q\right) \log \frac{p^{*}\left(y_{1}, y_{2} \mid u_{1}, u_{2}, q\right)}{p^{*}\left(y_{1} \mid u_{1}, u_{2}, q\right) p^{*}\left(y_{2} \mid u_{1}, u_{2}, q\right)} \\
& =\mathbb{E}_{p^{*}} \log o\left(Y_{1}, Y_{2}\right) p^{*}\left(Y_{1}, Y_{2} \mid U_{1}, U_{2}, Q\right) \\
& \quad-\mathbb{E}_{p^{*}} \log o\left(Y_{1}, Y_{2}\right) p^{*}\left(Y_{1} \mid U_{1}, U_{2}, Q\right) p^{*}\left(Y_{2} \mid U_{1}, U_{2}, Q\right) .
\end{aligned}
$$

Hence, we can interpret the auxiliary random variables corresponding to $p^{*}$ as optimal rate-limited side informations for both betting strategies. Moreover, optimal bets for each strategy are given by

1. $b^{*}\left(y_{1}, y_{2}\right)=p^{*}\left(y_{1}, y_{2} \mid u_{1}, u_{2}, q\right)$ for the joint-wager, and

2. $b_{1}^{*}\left(y_{1}\right)=p^{*}\left(y_{1} \mid u_{1}, u_{2}, q\right), b_{2}^{*}\left(y_{2}\right)=p^{*}\left(y_{2} \mid u_{1}, u_{2}, q\right)$ for the product-wager.

Since $\mathcal{P}\left(R_{1}, R_{2}\right) \subseteq \mathcal{P}\left(R_{1}^{\prime}, R_{2}^{\prime}\right)$ for $R_{1} \leq R_{1}^{\prime}$ and $R_{2} \leq R_{2}^{\prime}$, the function $\Delta\left(R_{1}, R_{2}\right)$ is nonincreasing in $R_{1}$ and $R_{2}$. Thus, the benefits of using the jointwager over the product-wager diminish in the amount of side-information available. It is also not difficult to show that $\Delta\left(R_{1}, R_{2}\right)$ is jointly convex in $\left(R_{1}, R_{2}\right)$.

Furthermore, for rate-pairs $\left(R_{1}, R_{2}\right)$ and $\left(R_{1}^{\prime}, R_{2}^{\prime}\right)$ satisfying $R_{1}<R_{1}^{\prime}$ and $R_{2}<R_{2}^{\prime}$, there exist corresponding optimal joint- and product-wagers $b^{*}\left(y_{1}, y_{2}\right)$ and $b_{1}^{*}\left(y_{1}\right) b_{2}^{*}\left(y_{2}\right)$, and $b^{*^{\prime}}\left(y_{1}, y_{2}\right)$ and $b_{1}^{*^{\prime}}\left(y_{1}\right) b_{2}^{*^{\prime}}\left(y_{2}\right)$, respectively, satisfying

$$
D\left(b^{*^{\prime}}\left(y_{1}, y_{2}\right) \| b_{1}^{*^{\prime}}\left(y_{1}\right) b_{2}^{*^{\prime}}\left(y_{2}\right)\right)<D\left(b^{*}\left(y_{1}, y_{2}\right) \| b_{1}^{*}\left(y_{1}\right) b_{2}^{*}\left(y_{2}\right)\right) .
$$

So, roughly speaking, the joint-wager and product-wager look "more alike" as the amount of side information is increased. The proof of the strict inequality in 111) can be inferred from the proof of Lemma 10 in Appendix $\mathrm{F}$.

To conclude this example, we note that $\Delta\left(R_{1}, R_{2}\right)$ enjoys a great deal of symmetry near the origin in the sense that side information from either encoder contributes approximately the same amount to the improvement of the productwager. We state this formally as a theorem:

Theorem 7. Define $\rho_{m}\left(Y_{1}, Y_{2}\right)$ to be the Hirschfeld-Gebelein-Rényi maximal correlation between random variables $Y_{1}$ and $Y_{2}$. Then, $\Delta\left(R_{1}, R_{2}\right) \geq I\left(Y_{1} ; Y_{2}\right)-$ $\rho_{m}^{2}\left(Y_{1}, Y_{2}\right) \cdot\left(R_{1}+R_{2}\right)$. Moreover, this bound is tight as $\left(R_{1}, R_{2}\right) \rightarrow(0,0)$.

Proof. If $R_{2}=0$, then it is readily verified that $\Delta\left(R_{1}, 0\right)$ can be expressed as follows:

$$
\Delta\left(R_{1}, 0\right)=I\left(Y_{1} ; Y_{2}\right)-\max _{\substack{p\left(u_{1} \mid y_{1}\right): I\left(U_{1} ; Y_{1}\right)=R_{1}, U_{1} \rightarrow Y_{1} \rightarrow Y_{2},\left|\mathcal{U}_{1}\right| \leq\left|\mathcal{Y}_{1}\right|+1}} I\left(U_{1} ; Y_{2}\right) .
$$

By symmetry:

$$
\Delta\left(0, R_{2}\right)=I\left(Y_{1} ; Y_{2}\right)-\max _{\substack{p\left(u_{2} \mid y_{2}\right): I\left(U_{2} ; Y_{2}\right)=R_{2}, U_{2} \rightarrow Y_{2} \rightarrow Y_{1},\left|\mathcal{U}_{2}\right| \leq\left|\mathcal{Y}_{2}\right|+1}} I\left(U_{2} ; Y_{1}\right) .
$$


Here, we can apply a result of Erkip [19, Theorem 10] to evaluate the gradient of $\Delta\left(R_{1}, R_{2}\right)$ at $\left(R_{1}, R_{2}\right)=(0,0)$ :

$$
\left.\frac{\partial}{\partial R_{1}} \Delta\left(R_{1}, R_{2}\right)\right|_{\left(R_{1}, R_{2}\right)=(0,0)}=\left.\frac{\partial}{\partial R_{2}} \Delta\left(R_{1}, R_{2}\right)\right|_{\left(R_{1}, R_{2}\right)=(0,0)}=-\rho_{m}^{2}\left(Y_{1}, Y_{2}\right) .
$$

Note, since $\Delta\left(R_{1}, 0\right)$ and $\Delta\left(0, R_{2}\right)$ are each convex in their respective variable and $\Delta(0,0)=I\left(Y_{1} ; Y_{2}\right)$, we have

$$
\begin{aligned}
& \Delta\left(R_{1}, 0\right) \geq I\left(Y_{1} ; Y_{2}\right)-\rho_{m}^{2}\left(Y_{1}, Y_{2}\right) R_{1} \\
& \Delta\left(0, R_{2}\right) \geq I\left(Y_{1} ; Y_{2}\right)-\rho_{m}^{2}\left(Y_{1}, Y_{2}\right) R_{2} .
\end{aligned}
$$

Taking this one step further, for $\nu_{1}, \nu_{2}>0$, we can evaluate the one-sided derivative:

$$
\lim _{\lambda \downarrow 0} \frac{\Delta\left(\lambda \nu_{1}, \lambda \nu_{2}\right)-\Delta(0,0)}{\lambda}=-\rho_{m}^{2}\left(Y_{1}, Y_{2}\right) \cdot\left(\nu_{1}+\nu_{2}\right) .
$$

We remark that (14) does not follow immediately from (12) since the point at which we are taking the derivatives (i.e., the origin) does not lie in an open neighborhood of the domain. Nonetheless, the expected result holds.

Since $\Delta\left(R_{1}, R_{2}\right)$ is convex, we obtain an upper bound on the one-sided derivative as follows:

$$
\begin{aligned}
\lim _{\lambda \downarrow 0} \frac{\Delta\left(\lambda \nu_{1}, \lambda \nu_{2}\right)-\Delta(0,0) \leq}{\lambda} \leq & \lim _{\lambda \downarrow 0} \frac{\frac{1}{2} \Delta\left(2 \lambda \nu_{1}, 0\right)+\frac{1}{2} \Delta\left(0,2 \lambda \nu_{2}\right)-\Delta(0,0 ; p)}{\lambda} \\
= & \frac{1}{2} \lim _{\lambda \downarrow 0} \frac{\Delta\left(\lambda 2 \nu_{1}, 0\right)-\Delta(0,0)}{\lambda} \\
& +\frac{1}{2} \lim _{\lambda \downarrow 0} \frac{\Delta\left(0, \lambda 2 \nu_{2}\right)-\Delta(0,0)}{\lambda} \\
= & -\rho_{m}^{2}\left(Y_{1}, Y_{2}\right) \cdot\left(\nu_{1}+\nu_{2}\right),
\end{aligned}
$$

where the final equality follows by 12 and the positive homogeneity of the directional derivative.

Therefore, to complete the proof of (14), it suffices to prove the lower bound

$$
\lim _{\lambda \downarrow 0} \frac{\Delta\left(\lambda \nu_{1}, \lambda \nu_{2}\right)-\Delta(0,0)}{\lambda} \geq-\rho_{m}^{2}\left(Y_{1}, Y_{2}\right) \cdot\left(\nu_{1}+\nu_{2}\right) .
$$


To this end, fix $\lambda, \nu_{1}, \nu_{2}>0$ and observe that

$$
\begin{aligned}
\frac{\Delta}{\Delta(}\left(\lambda \nu_{1}, \lambda \nu_{2}\right)-\Delta(0,0) & \lambda \\
= & \frac{1}{\lambda} \inf _{p \in \mathcal{P}\left(\lambda \nu_{1}, \lambda \nu_{2}\right)}\left\{I\left(Y_{1} ; Y_{2}\left|U_{1}, U_{2}\right| Q\right)-I\left(Y_{1} ; Y_{2}\right)\right\} \\
= & \frac{1}{\lambda} \inf _{p \in \mathcal{P}\left(\lambda \nu_{1}, \lambda \nu_{2}\right)}\left\{I\left(Y_{1}, Y_{2} ; U_{1}, U_{2} \mid Q\right)-I\left(Y_{1} ; U_{1}, U_{2} \mid Q\right)-I\left(Y_{2} ; U_{1}, U_{2} \mid Q\right)\right\} \\
= & \left(\nu_{1}+\nu_{2}\right)-\frac{1}{\lambda}\left(I_{p^{*}}\left(Y_{1} ; U_{1}, U_{2} \mid Q\right)+I_{p^{*}}\left(Y_{2} ; U_{1}, U_{2} \mid Q\right)\right) \\
= & \left(\nu_{1}+\nu_{2}\right)-\frac{1}{\lambda}\left(I_{p^{*}}\left(Y_{1} ; U_{1} \mid U_{2}, Q\right)+I_{p^{*}}\left(Y_{1} ; U_{2} \mid Q\right)\right. \\
& \left.\quad+I_{p^{*}}\left(Y_{2} ; U_{2} \mid U_{1}, Q\right)+I_{p^{*}}\left(Y_{2} ; U_{1} \mid Q\right)\right) \\
\geq & \left(\nu_{1}+\nu_{2}\right)-\rho_{m}^{2}\left(Y_{1}, Y_{2}\right)\left(2 \nu_{1}+2 \nu_{2}\right) \\
& \quad-\frac{\left(1-\rho_{m}^{2}\left(Y_{1}, Y_{2}\right)\right)}{\lambda}\left(I_{p^{*}}\left(Y_{1} ; U_{1} \mid U_{2}, Q\right)+I_{p^{*}}\left(Y_{2} ; U_{2} \mid U_{1}, Q\right)\right) \\
= & -\rho_{m}^{2}\left(Y_{1}, Y_{2}\right)\left(\nu_{1}+\nu_{2}\right)+\left(1-\rho_{m}^{2}\left(Y_{1}, Y_{2}\right)\right)\left(\nu_{1}+\nu_{2}\right) \\
& \quad-\frac{\left(1-\rho_{m}^{2}\left(Y_{1}, Y_{2}\right)\right)}{\lambda}\left(I_{p^{*}}\left(Y_{1} ; U_{1} \mid U_{2}, Q\right)+I_{p^{*}}\left(Y_{2} ; U_{2} \mid U_{1}, Q\right)\right) \\
\geq & -\rho_{m}^{2}\left(Y_{1}, Y_{2}\right)\left(\nu_{1}+\nu_{2}\right) .
\end{aligned}
$$

In the above string of inequalities

- 15 follows by definition of $\Delta\left(R_{1}, R_{2}\right)$.

- Equality 16 follows since Lemma 10 guarantees that the infimum is attained in 15 for some $p^{*} \in \mathcal{P}\left(\lambda \nu_{1}, \lambda \nu_{2}\right)$ satisfying $I_{p^{*}}\left(Y_{1}, Y_{2} ; U_{1}, U_{2} \mid Q\right)=$ $\lambda\left(\nu_{1}+\nu_{2}\right)$. Here, we write $I_{p^{*}}\left(Y_{1}, Y_{2} ; U_{1}, U_{2} \mid Q\right)$ to denote the mutual information $I\left(Y_{1}, Y_{2} ; U_{1}, U_{2} \mid Q\right)$ evaluated for the distribution $p^{*}$.

- To see that (17) holds, note that

$$
I_{p^{*}}\left(Y_{2} ; U_{2} \mid Q\right)=\lambda \nu_{1}+\lambda \nu_{2}-I_{p^{*}}\left(Y_{1} ; U_{1} \mid U_{2}, Q\right),
$$

and thus

$$
\begin{aligned}
& I\left(Y_{1} ; Y_{2}\right)-\rho_{m}^{2}\left(Y_{1}, Y_{2}\right)\left(\lambda \nu_{1}+\lambda \nu_{2}-I_{p^{*}}\left(Y_{1} ; U_{1} \mid U_{2}, Q\right)\right) \\
& \quad \leq \Delta\left(0, \lambda \nu_{1}+\lambda \nu_{2}-I_{p^{*}}\left(Y_{1} ; U_{1} \mid U_{2}, Q\right)\right) \\
& \quad=I\left(Y_{1} ; Y_{2}\right)-\max _{p\left(\tilde{u}_{2} \mid y_{2}\right): I\left(Y_{2} ; \tilde{U}_{2}\right) \leq \lambda \nu_{1}+\lambda \nu_{2}-I_{p^{*}}\left(Y_{1} ; U_{1} \mid U_{2}, Q\right),} I\left(\tilde{U}_{2} ; Y_{1}\right) \\
& \quad \leq I\left(Y_{1} ; Y_{2}\right)-I_{p^{*}}\left(Y_{1} ; U_{2} \mid Q\right),
\end{aligned}
$$

which implies

$$
-\rho_{m}^{2}\left(Y_{1}, Y_{2}\right)\left(\lambda \nu_{1}+\lambda \nu_{2}-I_{p^{*}}\left(Y_{1} ; U_{1} \mid U_{2}, Q\right)\right) \leq-I_{p^{*}}\left(Y_{1} ; U_{2} \mid Q\right) .
$$

The above steps are justified as follows: 
- 19) follows from 13.

- 20) follows by definition of the function $\Delta(0, x)$.

- 21) follows since $Q$ is independent of $Y_{1}, Y_{2}$ (by definition of $p^{*}$ ), and thus $\tilde{U}_{2}=\left(U_{2}, Q\right)$ lies in the set over which we take the maximum in 20.

By symmetry, we conclude that

$$
\begin{aligned}
- & \left(I_{p^{*}}\left(Y_{1} ; U_{2} \mid Q\right)+I_{p^{*}}\left(Y_{2} ; U_{1} \mid Q\right)\right) \\
& \geq-\rho_{m}^{2}\left(Y_{1}, Y_{2}\right)\left(2 \lambda \nu_{1}+2 \lambda \nu_{2}-I_{p^{*}}\left(Y_{1} ; U_{1} \mid U_{2}, Q\right)-I_{p^{*}}\left(Y_{2} ; U_{2} \mid U_{1}, Q\right)\right),
\end{aligned}
$$

and (17) follows.

- (18) follows since $\lambda \nu_{1} \geq I_{p^{*}}\left(Y_{1} ; U_{1} \mid U_{2}, Q\right)$ and $\lambda \nu_{2} \geq I_{p^{*}}\left(Y_{2} ; U_{2} \mid U_{1}, Q\right)$ for $p^{*} \in \mathcal{P}\left(\lambda \nu_{1}, \lambda \nu_{2}\right)$.

\subsection{An Application: List Decoding}

In the previous example, we did not take advantage of the stronger converse result which we proved in Appendix E (see the discussion in Section 4.3). In this section, we give an application that requires this strengthened result.

Formally, a 2-list code (of blocklength $n$ consists) of encoding functions:

$$
g_{i}^{(n)}: \mathcal{Y}_{i}^{n} \rightarrow\left\{1, \ldots, M_{i}^{(n)}\right\} \text { for } i=1,2
$$

and list decoding functions

$$
\begin{aligned}
& L_{1}^{(n)}:\left\{1, \ldots, M_{1}^{(n)}\right\} \times\left\{1, \ldots, M_{2}^{(n)}\right\} \rightarrow 2^{\mathcal{Y}_{1}^{n}} \\
& L_{2}^{(n)}:\left\{1, \ldots, M_{1}^{(n)}\right\} \times\left\{1, \ldots, M_{2}^{(n)}\right\} \rightarrow 2^{\mathcal{Y}_{2}^{n}} .
\end{aligned}
$$

A list decoding tuple $\left(R_{1}, R_{2}, \Delta_{1}, \Delta_{2}\right)$ is achievable if, for any $\epsilon>0$, there exists a 2-list code of blocklength $n$ satisfying the rate constraints

$$
\begin{aligned}
& \frac{1}{n} \log M_{1}^{(n)} \leq R_{1}+\epsilon \\
& \frac{1}{n} \log M_{2}^{(n)} \leq R_{2}+\epsilon,
\end{aligned}
$$

and the probability of list-decoding error constraints

$$
\begin{aligned}
& \operatorname{Pr}\left[Y_{1}^{n} \notin L_{1}^{(n)}\left(g_{1}^{(n)}\left(Y_{1}^{n}\right), g_{2}^{(n)}\left(Y_{2}^{n}\right)\right)\right] \leq \epsilon, \\
& \operatorname{Pr}\left[Y_{2}^{n} \notin L_{2}^{(n)}\left(g_{1}^{(n)}\left(Y_{1}^{n}\right), g_{2}^{(n)}\left(Y_{2}^{n}\right)\right)\right] \leq \epsilon .
\end{aligned}
$$


with list sizes

$$
\begin{aligned}
& \frac{1}{n} \log \left|L_{1}^{(n)}\right| \leq \Delta_{1}+\epsilon \\
& \frac{1}{n} \log \left|L_{2}^{(n)}\right| \leq \Delta_{2}+\epsilon .
\end{aligned}
$$

With a 2-list code so defined, the following theorem shows that the 2-list decoding problem and multiterminal source coding problem under logarithmic loss are equivalent (inasmuch as the achievable regions are identical):

Theorem 8. The list decoding tuple $\left(R_{1}, R_{2}, \Delta_{1}, \Delta_{2}\right)$ is achievable if and only if

$$
\begin{aligned}
R_{1} & \geq I\left(U_{1} ; Y_{1} \mid U_{2}, Q\right) \\
R_{2} & \geq I\left(U_{2} ; Y_{2} \mid U_{1}, Q\right) \\
R_{1}+R_{2} & \geq I\left(U_{1}, U_{2} ; Y_{1}, Y_{2} \mid Q\right) \\
\Delta_{1} & \geq H\left(Y_{1} \mid U_{1}, U_{2}, Q\right) \\
\Delta_{2} & \geq H\left(Y_{2} \mid U_{1}, U_{2}, Q\right) .
\end{aligned}
$$

for some joint distribution

$$
p\left(y_{1}, y_{2}, u_{1}, u_{2}, q\right)=p\left(y_{1}, y_{2}\right) p\left(u_{1} \mid y_{1}, q\right) p\left(u_{2} \mid y_{2}, q\right) p(q),
$$

where $\left|\mathcal{U}_{1}\right| \leq\left|\mathcal{Y}_{1}\right|,\left|\mathcal{U}_{2}\right| \leq\left|\mathcal{Y}_{2}\right|$, and $|\mathcal{Q}| \leq 5$.

Remark 2. We note that a similar connection to list decoding can be made for other multiterminal scenarios, in particular the CEO problem. $1]$ :

To prove the theorem, we require a slightly modified version of 20, Lemma

Lemma 2. If the list decoding tuple $\left(R_{1}, R_{2}, \Delta_{1}, \Delta_{2}\right)$ is achieved by a sequence of 2-list codes $\left\{g_{1}^{(n)}, g_{2}^{(n)}, L_{1}^{(n)}, L_{2}^{(n)}\right\}_{n \rightarrow \infty}$, then

$$
\begin{aligned}
& H\left(Y_{1}^{n} \mid g_{1}^{(n)}\left(Y_{1}^{n}\right), g_{2}^{(n)}\left(Y_{2}^{n}\right)\right) \leq\left|L_{1}^{(n)}\right|+n \epsilon_{n} \\
& H\left(Y_{2}^{n} \mid g_{1}^{(n)}\left(Y_{1}^{n}\right), g_{2}^{(n)}\left(Y_{2}^{n}\right)\right) \leq\left|L_{2}^{(n)}\right|+n \epsilon_{n}
\end{aligned}
$$

where $\epsilon_{n} \rightarrow 0$ as $n \rightarrow \infty$.

Proof. The proof is virtually identical to that of [20, Lemma 1], and is therefore omitted.

Proof of Theorem 8. First observe that the direct part is trivial. Indeed, for a joint distribution $p\left(y_{1}, y_{2}, u_{1}, u_{2}, q\right)=p\left(y_{1}, y_{2}\right) p\left(u_{1} \mid y_{1}, q\right) p\left(u_{2} \mid y_{2}, q\right) p(q)$, apply the Berger-Tung achievability scheme and take $L_{i}^{(n)}$ to be the set of $y_{i}^{n}$ sequences which are jointly typical with the decoded quantizations $\left(U_{1}^{n}, U_{2}^{n}\right)$. This set has cardinality no larger than $2^{n\left(H\left(Y_{i} \mid U_{1}, U_{2}, Q\right)+\epsilon\right)}$, which proves achievability. 
To see the converse, note that setting

$$
\hat{Y}_{i}^{n}=\operatorname{Pr}\left[Y_{i}^{n} \mid g_{1}^{(n)}\left(Y_{1}^{n}\right), g_{2}^{(n)}\left(Y_{2}^{n}\right)\right]
$$

achieves a logarithmic loss of $\frac{1}{n} H\left(Y_{i}^{n} \mid g_{1}^{(n)}\left(Y_{1}^{n}\right), g_{2}^{(n)}\left(Y_{2}^{n}\right)\right)$ for source $i$ in the setting where reproductions are not restricted to product distributions. Applying the strengthened converse of Theorem 6 together with Lemma 2 yields the desired result.

\section{Relationship to the General Multiterminal Source Coding Problem}

In this section, we relate our results for logarithmic loss to multiterminal source coding problems with arbitrary distortion measures and reproduction alphabets.

As before, we let $\left\{Y_{1, j}, Y_{2, j}\right\}_{j=1}^{n}$ be a sequence of $n$ independent, identically distributed random variables with finite alphabets $\mathcal{Y}_{1}$ and $\mathcal{Y}_{2}$, respectively, and joint $\operatorname{pmf} p\left(y_{1}, y_{2}\right)$.

In this section, the reproduction alphabets $\breve{\mathcal{Y}}_{i}, i=1,2$, are arbitrary. We also consider generic distortion measures:

$$
\breve{d}_{i}: \mathcal{Y}_{i} \times \breve{\mathcal{Y}}_{i} \rightarrow \mathbb{R}^{+} \text {for } i=1,2,
$$

where $\mathbb{R}^{+}$denotes the set of nonnegative real numbers. The sequence distortion is then defined as follows:

$$
\breve{d}_{i}\left(y_{i}^{n}, \breve{y}_{i}^{n}\right)=\frac{1}{n} \sum_{j=1}^{n} \breve{d}_{i}\left(y_{i, j}, \breve{y}_{i, j}\right)
$$

We will continue to let $d(\cdot, \cdot)$ and $\hat{\mathcal{Y}}_{1}, \hat{\mathcal{Y}}_{2}$ denote the logarithmic loss distortion measure and the associated reproduction alphabets, respectively.

A rate distortion code (of blocklength $n$ ) consists of encoding functions:

$$
\breve{g}_{i}^{(n)}: \mathcal{Y}_{i}^{n} \rightarrow\left\{1, \ldots, M_{i}^{(n)}\right\} \text { for } i=1,2
$$

and decoding functions

$$
\breve{\psi}_{i}^{(n)}:\left\{1, \ldots, M_{1}^{(n)}\right\} \times\left\{1, \ldots, M_{2}^{(n)}\right\} \rightarrow \breve{\mathcal{Y}}_{i}^{n} \text { for } i=1,2 .
$$

A rate distortion vector $\left(R_{1}, R_{2}, D_{1}, D_{2}\right)$ is strict-sense achievable if there exists a blocklength $n$, encoding functions $\breve{g}_{1}^{(n)}, \breve{g}_{2}^{(n)}$ and a decoder $\left(\breve{\psi}_{1}^{(n)}, \breve{\psi}_{2}^{(n)}\right)$ such that

$$
\begin{aligned}
& R_{i} \geq \frac{1}{n} \log M_{i}^{(n)} \text { for } i=1,2 \\
& D_{i} \geq \mathbb{E} \breve{d}_{i}\left(Y_{i}^{n}, \breve{Y}_{i}^{n}\right) \text { for } i=1,2 .
\end{aligned}
$$


Where

$$
\breve{Y}_{i}^{n}=\breve{\psi}_{i}^{(n)}\left(\breve{g}_{1}^{(n)}\left(Y_{1}^{n}\right), \breve{g}_{2}^{(n)}\left(Y_{2}^{n}\right)\right) \text { for } i=1,2 .
$$

For these functions, we define the quantity

$$
\beta_{i}\left(\breve{g}_{1}^{(n)}, \breve{g}_{2}^{(n)}, \breve{\psi}_{1}^{(n)}, \breve{\psi}_{2}^{(n)}\right):=\frac{1}{n} \sum_{j=1}^{n} \mathbb{E} \log \left(\sum_{y_{i} \in \mathcal{Y}_{i}} 2^{-\breve{d}_{i}\left(y_{i}, \breve{Y}_{i, j}\right)}\right) \text { for } i=1,2
$$

Now, let $\beta_{i}\left(R_{1}, R_{2}, D_{1}, D_{2}\right)$ be the infimum of the $\beta_{i}\left(\breve{g}_{1}^{(n)}, \breve{g}_{2}^{(n)}, \breve{\psi}_{1}^{(n)}, \breve{\psi}_{2}^{(n)}\right)$ 's, where the infimum is taken over all codes that achieve the rate distortion vector $\left(R_{1}, R_{2}, D_{1}, D_{2}\right)$.

At this point it is instructive to pause and consider some examples.

Example 1 (Binary Sources and Hamming Distortion). For $i=1,2$, let $\breve{\mathcal{Y}}_{i}=$ $\mathcal{Y}_{i}=\{0,1\}$ and let $\breve{d}_{i}$ be the $\alpha$-scaled Hamming distortion measure:

$$
\breve{d}_{i}\left(y_{i}, \breve{y}_{i}\right)= \begin{cases}0 & \text { if } \breve{y}_{i}=y_{i} \\ \alpha & \text { if } \breve{y}_{i} \neq y_{i}\end{cases}
$$

In this case,

$$
\sum_{y_{i} \in \mathcal{Y}_{i}} 2^{-\breve{d}_{i}\left(y_{i}, \breve{Y}_{i, j}\right)}=2^{0}+2^{-\alpha},
$$

so $\beta_{i}\left(R_{1}, R_{2}, D_{1}, D_{2}\right)=\log \left(1+2^{-\alpha}\right)$ for any $\left(R_{1}, R_{2}, D_{1}, D_{2}\right)$. This notion that $\beta_{i}\left(R_{1}, R_{2}, D_{1}, D_{2}\right)$ is a constant extends to all distortion measures for which the columns of the $\left|\mathcal{Y}_{i}\right| \times\left|\breve{Y}_{i}\right|$ distortion matrix are permutations of one another.

Example 2 (Binary Sources and Erasure Distortion). For $i=1,2$, let $\mathcal{Y}_{i}=$ $\{0,1\}, \breve{\mathcal{Y}}_{i}=\{0,1, e\}$ and let $\breve{d}_{i}$ be the standard erasure distortion measure:

$$
\breve{d}_{i}\left(y_{i}, \breve{y}_{i}\right)= \begin{cases}0 & \text { if } \breve{y}_{i}=y_{i} \\ 1 & \text { if } \breve{y}_{i}=e \\ \infty & \text { if } \breve{y}_{i} \in\{0,1\} \text { and } \breve{y}_{i} \neq y_{i} .\end{cases}
$$

In this case,

$$
\sum_{y_{i} \in \mathcal{Y}_{i}} 2^{-\breve{d}_{i}\left(y_{i}, \breve{Y}_{i, j}\right)}= \begin{cases}2^{-\infty}+2^{0}=1 & \text { if } \breve{Y}_{i, j} \in\{0,1\} \\ 2^{-1}+2^{-1}=1 & \text { if } \breve{Y}_{i, j}=e\end{cases}
$$

so $\beta_{i}\left(R_{1}, R_{2}, D_{1}, D_{2}\right)=0$ for any $\left(R_{1}, R_{2}, D_{1}, D_{2}\right)$. This result can easily be extended to erasure distortion on larger alphabets by setting the penalty to $\log \left|\mathcal{Y}_{i}\right|$ when $\breve{Y}_{i}=e$. 
Theorem 9. Suppose $\left(R_{1}, R_{2}, D_{1}, D_{2}\right)$ is strict-sense achievable for the general multiterminal source coding problem. Then

$$
\left.\begin{array}{rl}
R_{1} & \geq I\left(U_{1} ; Y_{1} \mid U_{2}, Q\right) \\
R_{2} & \geq I\left(U_{2} ; Y_{2} \mid U_{1}, Q\right) \\
R_{1}+R_{2} & \geq I\left(U_{1}, U_{2} ; Y_{1}, Y_{2} \mid Q\right) \\
D_{1} & \geq H\left(Y_{1} \mid U_{1}, U_{2}, Q\right)-\beta_{1}\left(R_{1}, R_{2}, D_{1}, D_{2}\right) \\
D_{2} & \geq H\left(Y_{2} \mid U_{1}, U_{2}, Q\right)-\beta_{2}\left(R_{1}, R_{2}, D_{1}, D_{2}\right)
\end{array}\right\}
$$

for some joint distribution $p\left(y_{1}, y_{2}\right) p(q) p\left(u_{1} \mid y_{1}, q\right) p\left(u_{2} \mid y_{2}, q\right)$ with $\left|\mathcal{U}_{i}\right| \leq\left|\mathcal{Y}_{i}\right|$ and $|\mathcal{Q}| \leq 5$.

Proof. Since $\left(R_{1}, R_{2}, D_{1}, D_{2}\right)$ is strict-sense achievable, there exists a blocklength $n$, encoding functions $\breve{g}_{1}^{(n)}, \breve{g}_{2}^{(n)}$ and a decoder $\left(\breve{\psi}_{1}^{(n)}, \breve{\psi}_{2}^{(n)}\right)$ satisfying (22)(23). Given these functions, the decoder can generate reproductions $\breve{Y}_{1}^{n}, \breve{Y}_{2}^{n}$ satisfying the average distortion constraints 23). From the reproduction $\breve{Y}_{i}^{n}$, we construct the reproduction $\hat{Y}_{i}^{n}$ as follows:

$$
\hat{Y}_{j}\left(y_{i}\right)=\frac{2^{-\breve{d}_{i}\left(y_{i}, \breve{Y}_{i, j}\right)}}{\sum_{y_{i}^{\prime} \in \mathcal{Y}_{i}} 2^{-\breve{d}_{i}\left(y_{i}^{\prime}, \breve{Y}_{i, j}\right)}} .
$$

Now, using the logarithmic loss distortion measure, observe that $\hat{Y}_{i}^{n}$ satisfies

$$
\begin{aligned}
\mathbb{E} d\left(Y_{i}^{n}, \hat{Y}_{i}^{n}\right) & =\frac{1}{n} \sum_{j=1}^{n} \mathbb{E} \log \left(2^{\breve{d}_{i}\left(Y_{i, j}, \breve{Y}_{i, j}\right)}\right)+\frac{1}{n} \sum_{j=1}^{n} \mathbb{E} \log \left(\sum_{y_{i}^{\prime} \in \mathcal{Y}_{i}} 2^{-\breve{d}_{i}\left(y_{i}^{\prime}, \breve{Y}_{i, j}\right)}\right) \\
& =\frac{1}{n} \sum_{j=1}^{n} \mathbb{E} \breve{d}_{i}\left(Y_{i, j}, \breve{Y}_{i, j}\right)+\beta_{i}\left(\breve{g}_{1}^{(n)}, \breve{g}_{2}^{(n)}, \breve{\psi}_{1}^{(n)}, \breve{\psi}_{2}^{(n)}\right) \\
& \leq D_{i}+\beta_{i}\left(\breve{g}_{1}^{(n)}, \breve{g}_{2}^{(n)}, \breve{\psi}_{1}^{(n)}, \breve{\psi}_{2}^{(n)}\right) \\
& :=\tilde{D}_{i}
\end{aligned}
$$

Thus, $\left(R_{1}, R_{2}, \tilde{D}_{1}, \tilde{D}_{2}\right)$ is achievable for the multiterminal source coding problem with the logarithmic loss distortion measure. Applying Theorem 6 and taking the infimum over all coding schemes that achieve $\left(R_{1}, R_{2}, D_{1}, D_{2}\right)$ proves the theorem.

This outer bound is interesting because the region is defined over the same set of probability distributions that define the Berger-Tung inner bound. While the $\beta_{i}$ 's can be difficult to compute in general, we have shown that they can be readily determined for many popular distortion measures. As an application, we now give a quantitative approximation of the rate distortion region for binary sources subject to Hamming distortion constraints. Before proceeding, we prove the following lemma. 
Lemma 3. Suppose $\left(R_{1}, R_{2}, \tilde{D}_{1}, \tilde{D}_{2}\right)$ is strict-sense achievable for the multiterminal source coding problem with binary sources and $\breve{d}_{i}$ equal to the $\alpha_{i}$-scaled Hamming distortion measure, for $i=1,2$. Then the Berger-Tung achievability scheme can achieve a point $\left(R_{1}, R_{2}, D_{1}, D_{2}\right)$ satisfying

$$
D_{i}-\tilde{D}_{i} \leq\left(\frac{\alpha_{i}}{2}-1\right) H_{i}+\log \left(1+2^{-\alpha_{i}}\right)
$$

for some $H_{i} \in[0,1], i=1,2$.

Proof. By Theorem 9, $\left(R_{1}, R_{2}, \tilde{D}_{1}, \tilde{D}_{2}\right)$ satisfy (27) for some joint distribution $p\left(y_{1}, y_{2}\right) p(q) p\left(u_{1} \mid y_{1}, q\right) p\left(u_{2} \mid y_{2}, q\right)$. For this distribution, define the reproduction functions

$$
\breve{Y}_{i}\left(U_{1}, U_{2}, Q\right)=\arg \max _{y_{i}} p\left(y_{i} \mid U_{1}, U_{2}, Q\right) \text { for } i=1,2 \text {. }
$$

Then, observe that for $i=1,2$ :

$$
\begin{aligned}
\mathbb{E} \breve{d}_{i}\left(Y_{i}, \breve{Y}_{i}\right) & =\sum_{u_{1}, u_{2}, q} p\left(u_{1}, u_{2}, q\right)\left[\alpha_{i} \cdot \min _{y_{i}} p\left(y_{i} \mid u_{1}, u_{2}, q\right)+0 \cdot \max _{y_{i}} p\left(y_{i} \mid u_{1}, u_{2}, q\right)\right] \\
& =\alpha_{i} \sum_{u_{1}, u_{2}, q} p\left(u_{1}, u_{2}, q\right) \cdot \min _{y_{i}} p\left(y_{i} \mid u_{1}, u_{2}, q\right) \\
& \leq \frac{\alpha_{i}}{2} \sum_{u_{1}, u_{2}, q} p\left(u_{1}, u_{2}, q\right) \cdot H\left(Y_{i} \mid U_{1}, U_{2}, Q=u_{1}, u_{2}, q\right) \\
& =\frac{\alpha_{i}}{2} H\left(Y_{i} \mid U_{1}, U_{2}, Q\right) .
\end{aligned}
$$

Where 290 follows from the fact that $2 p \leq h_{2}(p)$ for $0 \leq p \leq 0.5$. Thus, $D_{i}=\frac{\alpha_{i}}{2} H\left(Y_{i} \mid U_{1}, U_{2}, Q\right)$ is achievable for rates $\left(R_{1}, R_{2}\right)$ using the Berger-Tung achievability scheme. Combining this with the fact that $\tilde{D}_{i} \geq H\left(Y_{i} \mid U_{1}, U_{2}, Q\right)-$ $\log \left(1+2^{-\alpha_{i}}\right)$, we see that

$$
D_{i}-\tilde{D}_{i} \leq \frac{\alpha_{i}}{2} H\left(Y_{i} \mid U_{1}, U_{2}, Q\right)-H\left(Y_{i} \mid U_{1}, U_{2}, Q\right)+\log \left(1+2^{-\alpha_{i}}\right) .
$$

Lemma 3 allows us to give a quantitative outer bound on the achievable rate distortion region in terms of the Berger-Tung inner bound.

Corollary 1. Suppose $\left(R_{1}, R_{2}, \tilde{D}_{1}^{(1)}, \tilde{D}_{2}^{(1)}\right)$ is strict-sense achievable for the multiterminal source coding problem with binary sources and $\breve{d}_{i}$ equal to the standard 1-scaled Hamming distortion measure, for $i=1,2$. Then the Berger-Tung achievability scheme can achieve a point $\left(R_{1}, R_{2}, D_{1}^{(1)}, D_{2}^{(1)}\right)$, where

$$
D_{i}^{(1)}-\tilde{D}_{i}^{(1)} \leq \frac{1}{2} \log \left(\frac{5}{4}\right)<0.161 \text { for } i=1,2 .
$$


Proof. For rates $\left(R_{1}, R_{2}\right)$, note that distortions $\left(\tilde{D}_{1}, \tilde{D}_{2}\right)$ are strict-sense achievable for the $\alpha_{i}$-scaled Hamming distortion measures if and only if distortions $\left(\tilde{D}_{1}^{(1)}, \tilde{D}_{2}^{(1)}\right)=\left(\frac{1}{\alpha_{1}} \tilde{D}_{1}, \frac{1}{\alpha_{2}} \tilde{D}_{2}\right)$ are strict-sense achievable for the 1-scaled Hamming distortion measure. Likewise, the point $\left(R_{1}, R_{2}, D_{1}, D_{2}\right)$ is achieved by the Berger-Tung coding scheme for the $\alpha_{i}$-scaled Hamming distortion measures if and only if $\left(R_{1}, R_{2}, \frac{1}{\alpha_{1}} D_{1}, \frac{1}{\alpha_{2}} D_{2}\right)$ is achieved by the Berger-Tung coding scheme for the 1-scaled Hamming distortion measure.

Thus, applying Lemma 3 , we can use the Berger-Tung achievability scheme to achieve a point $\left(R_{1}, R_{2}, D_{1}^{(1)}, D_{2}^{(1)}\right)$ satisfying

$$
\begin{aligned}
D_{i}^{(1)}-\tilde{D}_{i}^{(1)} & =\frac{1}{\alpha_{i}}\left(D_{i}-\tilde{D}_{i}\right) \\
& \leq \frac{1}{\alpha_{i}}\left(\frac{\alpha_{i}}{2}-1\right) H_{i}+\frac{1}{\alpha_{i}} \log \left(1+2^{-\alpha_{i}}\right) \\
& =\left(\frac{1}{2}-\frac{1}{\alpha_{i}}\right) H_{i}+\frac{1}{\alpha_{i}} \log \left(1+2^{-\alpha_{i}}\right)
\end{aligned}
$$

for some $H_{i} \in[0,1]$. We can optimize (30) over $\alpha_{i}$ to find the minimum gap for a given $H_{i}$. Maximizing over $H_{i} \in[0,1]$ then gives the worst-case gap. Straightforward calculus yields the saddle-point:

$$
\begin{aligned}
\max _{H_{i} \in[0,1]} & \inf _{\alpha_{i}>0}\left\{\left(\frac{1}{2}-\frac{1}{\alpha_{i}}\right) H_{i}+\frac{1}{\alpha_{i}} \log \left(1+2^{-\alpha_{i}}\right)\right\} \\
& =\inf _{\alpha_{i}>0} \max _{H_{i} \in[0,1]}\left\{\left(\frac{1}{2}-\frac{1}{\alpha_{i}}\right) H_{i}+\frac{1}{\alpha_{i}} \log \left(1+2^{-\alpha_{i}}\right)\right\} \\
& =\frac{1}{2} \log \left(\frac{5}{4}\right)<0.161,
\end{aligned}
$$

which is achieved for $\alpha_{i}=2$ and any $H \in[0,1]$.

Remark 3. We note briefly that this estimate can potentially be improved if one knows more about the source distribution.

\section{Concluding Remarks}

One immediate direction for further work would be to extend our results to more than two encoders. For the CEO problem, our results can be extended to an arbitrary number of encoders. This extension is proved in Appendix B.

On the other hand, generalizing the results for the two-encoder source coding problem with distortion constraints on $Y_{1}$ and $Y_{2}$ poses a significant challenge. The obvious point of difficulty in the proof is extending the interpolation argument to higher dimensions so that it yields a distribution with the desired properties. In fact, a "quick-fix" to the interpolation argument alone would not be sufficient since this would imply that the Berger-Tung inner bound is tight for more than two encoders. This is known to be false (even for the logarithmic loss distortion measure) since the Berger-Tung achievability scheme is not optimal for the lossless modulo-sum problem studied by Körner and Marton in [21]. 


\section{Acknowledgement}

The authors would like to thank Professors Suhas Diggavi and Aaron Wagner for the helpful discussions on this topic.

\section{A Cardinality Bounds on Auxiliary Random Variables}

In order to obtain tight cardinality bounds on the auxiliary random variables used throughout this paper, we refer to a recent result by Jana. In [17, the author carefully applies the Caratheodory-Fenchel-Eggleston theorem in order to obtain tight cardinality bounds on the auxiliary random variables in the Berger-Tung inner bound. This result extends the results and techniques employed by $\mathrm{Gu}$ and Effros for the Wyner-Ahlswede-Körner problem [22], and by $\mathrm{Gu}$, Jana, and Effros for the Wyner-Ziv problem [23. We now state Jana's result, appropriately modified for our purposes:

Consider an arbitrary joint distribution $p\left(v, y_{1}, \ldots, y_{m}\right)$ with random variables $V, Y_{1}, \ldots, Y_{m}$ coming from alphabets $\mathcal{V}, \mathcal{Y}_{1}, \ldots, \mathcal{Y}_{m}$ respectively.

Let $d_{l}: \mathcal{V} \times \hat{\mathcal{V}}_{l} \rightarrow \mathbb{R}, 1 \leq l \leq L$ be arbitrary distortion measures defined for possibly different reproduction alphabets $\hat{\mathcal{V}}_{l}$.

Definition 6. Define $\mathcal{A}^{\star}$ to be the set of $(m+L)$-vectors $\left(R_{1}, \ldots, R_{m}, D_{1}, \ldots, D_{L}\right)$ satisfying the following conditions:

1. auxiliary random variables $U_{1}, \ldots, U_{m}$ exist such that

$$
\sum_{i \in \mathcal{I}} R_{i} \geq I\left(Y_{\mathcal{I}} ; U_{\mathcal{I}} \mid U_{\mathcal{I}^{c}}\right), \text { for all } \mathcal{I} \subseteq\{1, \ldots, m\} \text {, and }
$$

2. mappings $\psi_{l}: \mathcal{U}_{1} \times \cdots \times \mathcal{U}_{m} \rightarrow \hat{\mathcal{V}}_{l}, 1 \leq l \leq L$ exist such that

$$
\mathbb{E} d_{l}\left(V, \psi_{l}\left(U_{1}, \ldots, U_{m}\right)\right) \leq D_{l}
$$

for some joint distribution

$$
p\left(v, y_{1}, \ldots, y_{m}\right) \prod_{j=1}^{m} p\left(u_{j} \mid y_{j}\right) .
$$

Lemma 4 (Lemma 2.2 from [17]). Every extreme point of $\mathcal{A}^{\star}$ corresponds to some choice of auxiliary variables $U_{1}, \ldots, U_{m}$ with alphabet sizes $\left|\mathcal{U}_{j}\right| \leq\left|\mathcal{Y}_{j}\right|$, $1 \leq j \leq m$.

In order to obtain the cardinality bounds for the CEO problem, we simply let $L=1, V=X$, and $\hat{\mathcal{V}}_{1}=\hat{\mathcal{X}}$. Defining

$$
d_{1}(x, \hat{x})=\log \left(\frac{1}{\hat{x}(x)}\right),
$$


we see that $\overline{\mathcal{R D}}_{C E O}^{\star}=\operatorname{conv}\left(\mathcal{A}^{\star}\right)$, where $\operatorname{conv}\left(\mathcal{A}^{\star}\right)$ denotes the convex hull of $\mathcal{A}^{\star}$. Therefore, Lemma 4 implies that all extreme points of $\overline{\mathcal{R D}}_{C E O}^{\star}$ are achieved with a choice of auxiliary random variables $U_{1}, \ldots, U_{m}$ with alphabet sizes $\left|\mathcal{U}_{j}\right| \leq\left|\mathcal{Y}_{j}\right|, 1 \leq j \leq m$. By timesharing between extreme points, any point in $\overline{\mathcal{R D}}_{C E O}^{\star}$ can be achieved for these alphabet sizes.

Obtaining the cardinality bounds for the multiterminal source coding problem proceeds in a similar fashion. In particular, let $L=m=2, V=\left(Y_{1}, Y_{2}\right)$, and $\hat{\mathcal{V}}_{j}=\hat{\mathcal{Y}}_{j}, j=1,2$. Defining

$$
d_{j}\left(\left(y_{1}, y_{2}\right), \hat{y}_{j}\right)=\log \left(\frac{1}{\hat{y}_{j}\left(y_{j}\right)}\right) \text { for } j=1,2,
$$

we see that $\overline{\mathcal{R D}}^{\star}=\operatorname{conv}\left(\mathcal{A}^{\star}\right)$. In this case, Lemma 4 implies that all extreme points of $\overline{\mathcal{R D}}^{\star}$ are achieved with a choice of auxiliary random variables $U_{1}, U_{2}$ with alphabet sizes $\left|\mathcal{U}_{j}\right| \leq\left|\mathcal{Y}_{j}\right|, 1 \leq j \leq 2$. By timesharing between extreme points, any point in $\overline{\mathcal{R D}}^{\star}$ can be achieved for these alphabet sizes.

In order to obtain cardinality bounds on the timesharing variable $Q$, we can apply Caratheodory's theorem (cf. 24]). In particular, if $C \subset \mathbb{R}^{n}$ is compact, then any point in $\operatorname{conv}(C)$ is a convex combination of at most $n+1$ points of $C$. Taking $C$ to be the closure of the set of extreme points of $\mathcal{A}^{\star}$ is sufficient for our purposes (boundedness of $C$ can be dealt with by a standard truncation argument).

\section{B Extension of CEO Results to $m$ Encoders}

In this appendix, we prove the generalization of Theorem 3 to $m$ encoders, which essentially amounts to extending the argument in the proof of Theorem 3 to the general case. We begin by stating the $m$-encoder generalizations of Theorems 1 and 2 the proofs of which are trivial extensions of the proofs given for the two-encoder case and are therefore omitted.

Definition 7. Let $\mathcal{R}_{C E O, m}^{i}$ be the set of all $\left(R_{1}, \ldots, R_{m}, D\right)$ satisfying

$$
\begin{aligned}
\sum_{i \in \mathcal{I}} R_{i} & \geq I\left(Y_{\mathcal{I}} ; U_{\mathcal{I}} \mid U_{\mathcal{I}^{c}}, Q\right) \text { for all } \mathcal{I} \subseteq\{1, \ldots, m\} \\
D & \geq H\left(X \mid U_{1}, \ldots, U_{m}, Q\right) .
\end{aligned}
$$

for some joint distribution $p(q) p(x) \prod_{i=1}^{m} p\left(y_{i} \mid x\right) p\left(u_{i} \mid y_{i}, q\right)$.

Theorem 10. All rate distortion vectors $\left(R_{1}, \ldots, R_{m}, D\right) \in \mathcal{R}_{C E O, m}^{i}$ are achievable.

Definition 8. Let $\mathcal{R}_{C E O, m}^{o}$ be the set of $\left(R_{1}, \ldots, R_{m}, D\right)$ satisfying

$$
\begin{aligned}
\sum_{i \in \mathcal{I}} R_{i} & \geq \sum_{i \in \mathcal{I}} I\left(U_{i} ; Y_{i} \mid X, Q\right)+H\left(X \mid U_{\mathcal{I}^{c}}, Q\right)-D \text { for all } \mathcal{I} \subseteq\{1, \ldots, m\} \\
D & \geq H\left(X \mid U_{1}, \ldots, U_{m}, Q\right) .
\end{aligned}
$$


for some joint distribution $p(q) p(x) \prod_{i=1}^{m} p\left(y_{i} \mid x\right) p\left(u_{i} \mid y_{i}, q\right)$.

Theorem 11. If $\left(R_{1}, \ldots, R_{m}, D\right)$ is strict-sense achievable, then $\left(R_{1}, \ldots, R_{m}, D\right) \in \mathcal{R}_{C E O, m}^{o}$.

Given the definitions of $\mathcal{R}_{C E O, m}^{i}$ and $\mathcal{R}_{C E O, m}^{o}$, the generalization of Theorem 3 to $m$ encoders is an immediate consequence of the following lemma:

Lemma 5. $\mathcal{R}_{C E O, m}^{o} \subseteq \mathcal{R}_{C E O, m}^{i}$.

Proof. Suppose $\left(R_{1}, \ldots, R_{m}, D\right) \in \mathcal{R}_{C E O, m}^{o}$, then by definition there exists $p(q)$ and conditional distributions $\left\{p\left(u_{i} \mid y_{i}, q\right)\right\}_{i=1}^{m}$ so that (31) and (32) are satisfied. For the joint distribution corresponding to $p(q)$ and conditional distributions $p\left\{\left(u_{i} \mid y_{i}, q\right)\right\}_{i=1}^{m}$, define $\mathcal{P}_{D} \subset \mathbb{R}^{m}$ to be the polytope defined by the inequalities 31. Now, to show $\left(R_{1}, \ldots, R_{m}, D\right) \in \mathcal{R}_{C E O, m}^{i}$, it suffices to show that each extreme point of $\mathcal{P}_{D}$ is dominated by a point in $\mathcal{R}_{C E O, m}^{i}$ that achieves distortion at most $D$.

To this end, define the set function $f: 2^{[m]} \rightarrow \mathbb{R}$ as follows:

$$
\begin{aligned}
f(\mathcal{I}) & :=I\left(Y_{\mathcal{I}} ; U_{\mathcal{I}} \mid U_{\mathcal{I}^{c}}, Q\right)-\left(D-H\left(X \mid U_{1}, \ldots, U_{m}, Q\right)\right) \\
& =\sum_{i \in \mathcal{I}} I\left(U_{i} ; Y_{i} \mid X, Q\right)+H\left(X \mid U_{\mathcal{I}^{c}}, Q\right)-D .
\end{aligned}
$$

It can be verified that the function $f$ and the function $f^{+}(\mathcal{I})=\max \{f(\mathcal{I}), 0\}$ are supermodular functions (see Appendix C). By construction, $\mathcal{P}_{D}$ is equal to the set of $\left(R_{1}, \ldots, R_{m}\right)$ which satisfy:

$$
\sum_{i \in \mathcal{I}} R_{i} \geq f^{+}(\mathcal{I})
$$

It follows by basic results in submodular optimization (see Appendix C) that, for a linear ordering $i_{1} \prec i_{2} \prec \cdots \prec i_{m}$ of $\{1, \ldots, m\}$, an extreme point of $\mathcal{P}_{D}$ can be greedily computed as follows:

$$
\tilde{R}_{i_{j}}=f^{+}\left(\left\{i_{1}, \ldots, i_{j}\right\}\right)-f^{+}\left(\left\{i_{1}, \ldots, i_{j-1}\right\}\right) \text { for } j=1, \ldots, m .
$$

Furthermore, all extreme points of $\mathcal{P}_{D}$ can be enumerated by looking over all linear orderings $i_{1} \prec i_{2} \prec \cdots \prec i_{m}$ of $\{1, \ldots, m\}$. Each ordering of $\{1, \ldots, m\}$ is analyzed in the same manner, hence we assume (for notational simplicity) that the ordering we consider is the natural ordering $i_{j}=j$.

Let $j$ be the first index for which $\tilde{R}_{j}>0$. Then, by construction,

$$
\tilde{R}_{k}=I\left(U_{k} ; Y_{k} \mid U_{k+1}, \ldots, U_{m}, Q\right) \text { for all } k>j .
$$

Furthermore, we must have $f\left(\left\{1, \ldots, j^{\prime}\right\}\right) \leq 0$ for all $j^{\prime}<j$. Thus, $\tilde{R}_{j}$ can be expressed as

$$
\begin{aligned}
\tilde{R}_{j} & =\sum_{i=1}^{j} I\left(Y_{i} ; U_{i} \mid X, Q\right)+H\left(X \mid U_{j+1}, \ldots, U_{m}, Q\right)-D \\
& =I\left(Y_{j} ; U_{j} \mid U_{j+1}, \ldots, U_{m}, Q\right)+f(\{1, \ldots, j-1\}) \\
& =(1-\theta) I\left(Y_{j} ; U_{j} \mid U_{j+1}, \ldots, U_{m}, Q\right)
\end{aligned}
$$


where $\theta \in[0,1)$ is defined as:

$$
\begin{aligned}
\theta & =\frac{-f(\{1, \ldots, j-1\})}{I\left(Y_{j} ; U_{j} \mid U_{j+1}, \ldots, U_{m}, Q\right)} \\
& =\frac{D-H\left(X \mid U_{1}, \ldots, U_{m}, Q\right)-I\left(U_{1}, \ldots, U_{j-1} ; Y_{1}, \ldots, Y_{j-1} \mid U_{j}, \ldots, U_{m}, Q\right)}{I\left(Y_{j} ; U_{j} \mid U_{j+1}, \ldots, U_{m}, Q\right)}
\end{aligned}
$$

By the results of Theorem 10 the rates $\left(\tilde{R}_{1}, \ldots, \tilde{R}_{m}\right)$ permit the following coding scheme: For a fraction $(1-\theta)$ of the time, a codebook can be used that allows the decoder to recover $U_{j}^{n}, \ldots, U_{m}^{n}$ with high probability. The other fraction $\theta$ of the time, a codebook can be used that allows the decoder to recover $U_{j+1}^{n}, \ldots, U_{m}^{n}$ with high probability. As $n \rightarrow \infty$, this coding scheme can achieve distortion

$$
\begin{aligned}
\tilde{D}= & (1-\theta) H\left(X \mid U_{j}, \ldots, U_{m}, Q\right)+\theta H\left(X \mid U_{j+1}, \ldots, U_{m}, Q\right) \\
= & H\left(X \mid U_{j}, \ldots, U_{m}, Q\right)+\theta I\left(X ; U_{j} \mid U_{j+1}, \ldots, U_{m}, Q\right) \\
= & H\left(X \mid U_{j}, \ldots, U_{m}, Q\right)+\frac{I\left(X ; U_{j} \mid U_{j+1}, \ldots, U_{m}, Q\right)}{I\left(Y_{j} ; U_{j} \mid U_{j+1}, \ldots, U_{m}, Q\right)} \times \\
& {\left[D-H\left(X \mid U_{1}, \ldots, U_{m}, Q\right)-I\left(U_{1}, \ldots, U_{j-1} ; Y_{1}, \ldots, Y_{j-1} \mid U_{j}, \ldots, U_{m}, Q\right)\right] } \\
\leq & H\left(X \mid U_{j}, \ldots, U_{m}, Q\right)+D-H\left(X \mid U_{1}, \ldots, U_{m}, Q\right) \\
& -I\left(U_{1}, \ldots, U_{j-1} ; Y_{1}, \ldots, Y_{j-1} \mid U_{j}, \ldots, U_{m}, Q\right) \\
= & D+I\left(X ; U_{1}, \ldots U_{j-1} \mid U_{j}, \ldots, U_{m}, Q\right) \\
& -I\left(U_{1}, \ldots, U_{j-1} ; Y_{1}, \ldots, Y_{j-1} \mid U_{j}, \ldots, U_{m}, Q\right) \\
= & D-I\left(U_{1}, \ldots, U_{j-1} ; Y_{1}, \ldots, Y_{j-1} \mid X, U_{j}, \ldots, U_{m}, Q\right) \\
\leq & D
\end{aligned}
$$

In the preceding string of inequalities $(33)$ follows since $U_{j}$ is conditionally independent of everything else given $\left(Y_{j}, Q\right)$, and (34) follows from the nonnegativity of mutual information.

Therefore, for every extreme point $\left(\tilde{R}_{1}, \ldots, \tilde{R}_{m}\right)$ of $\mathcal{P}_{D}$, the point $\left(\tilde{R}_{1}, \ldots, \tilde{R}_{m}, D\right)$ lies in $\mathcal{R}_{C E O, m}^{i}$. This proves the lemma.

Finally, we remark that the results of Appendix A imply that it suffices to consider auxiliary random variables $U_{1}, \ldots, U_{m}$ with alphabet sizes $\left|\mathcal{U}_{j}\right| \leq\left|\mathcal{Y}_{j}\right|$, $1 \leq j \leq m$. The timesharing variable $Q$ requires an alphabet size bounded by $|\mathcal{Q}| \leq m+2$.

\section{Supermodular Functions}

In this appendix, we review some basic results in submodular optimization that were used in Appendix B to prove Lemma 5. We tailor our statements toward supermodularity, since this is the property we require in Appendix $B$.

We begin by defining a supermodular function. 
Definition 9. Let $E=\{1, \ldots, n\}$ be a finite set. A function $s: 2^{E} \rightarrow \mathbb{R}$ is supermodular if for all $S, T \subseteq E$

$$
s(S)+s(T) \leq s(S \cap T)+s(S \cup T) .
$$

One of the fundamental results in submodular optimization is that a greedy algorithm minimizes a linear function over a supermodular polyhedron. By varying the linear function to be minimized, all extreme points of the supermodular polyhedron can be enumerated. In particular, define the supermodular polyhedron $\mathcal{P}(s) \subset \mathbb{R}^{n}$ to be the set of $x \in \mathbb{R}^{n}$ satisfying

$$
\sum_{i \in T} x_{i} \geq s(T) \text { for all } T \subseteq E .
$$

The following theorem provides an algorithm that enumerates the extreme points of $\mathcal{P}(s)$.

Theorem 12 (See 25 27]). For a linear ordering $e_{1} \prec e_{2} \prec \cdots \prec e_{n}$ of the elements in E, Algorithm C.1 returns an extreme point $v$ of $\mathcal{P}(s)$. Moreover, all extreme points of $\mathcal{P}(s)$ can be enumerated by considering all linear orderings of the elements of $E$.

Algorithm C.1: $\operatorname{Greedy}(s, E, \prec)$

comment: Returns extreme point $v$ of $\mathcal{P}(s)$ corresponding to the ordering $\prec$.

for $i=1, \ldots n$

Set $v_{i}=s\left(\left\{e_{1}, e_{2}, \ldots, e_{i}\right\}\right)-s\left(\left\{e_{1}, e_{2}, \ldots, e_{i-1}\right\}\right)$

return $(v)$

Proof. See 25 27.

Theorem 12 is the key tool we employ to establish Lemma 5 . In order to apply it, we require the following lemma.

Lemma 6. For any joint distribution of the form $p(q) p(x) \prod_{i=1}^{m} p\left(y_{i} \mid x\right) p\left(u_{i} \mid y_{i}, q\right)$ and fixed $D \in \mathbb{R}$, define the set function $f: 2^{[m]} \rightarrow \mathbb{R}$ as:

$$
\begin{aligned}
f(\mathcal{I}) & :=I\left(Y_{\mathcal{I}} ; U_{\mathcal{I}} \mid U_{\mathcal{I}^{c}}, Q\right)-\left(D-H\left(X \mid U_{1}, \ldots, U_{m}, Q\right)\right) \\
& =\sum_{i \in \mathcal{I}} I\left(U_{i} ; Y_{i} \mid X, Q\right)+H\left(X \mid U_{\mathcal{I}^{c}}, Q\right)-D,
\end{aligned}
$$

and the corresponding non-negative set function $f^{+}: 2^{[m]} \rightarrow \mathbb{R}$ as $f^{+}=$ $\max \{f, 0\}$. The functions $f$ and $f^{+}$are supermodular. 
Proof. In order to verify that $f$ is supermodular, it suffices to check that the function $f^{\prime}(\mathcal{I})=I\left(Y_{\mathcal{I}} ; U_{\mathcal{I}} \mid U_{\mathcal{I}^{c}}, Q\right)$ is supermodular since the latter two terms in (36) are constant. To this end, consider sets $T, S \subseteq\{1, \ldots, m\}$ and observe that:

$$
\begin{aligned}
f^{\prime}(S)+f^{\prime}(T)= & I\left(Y_{S} ; U_{S} \mid U_{S^{c}}, Q\right)+I\left(Y_{T} ; U_{T} \mid U_{T^{c}}, Q\right) \\
= & H\left(U_{S} \mid U_{S^{c}}, Q\right)-H\left(U_{S} \mid Y_{S}, Q\right)+H\left(U_{T} \mid U_{T^{c}}, Q\right)-H\left(U_{T} \mid Y_{T}, Q\right) \\
= & H\left(U_{S} \mid U_{S^{c}}, Q\right)+H\left(U_{T} \mid U_{T^{c}}, Q\right) \\
& -H\left(U_{S \cup T} \mid Y_{S \cup T}, Q\right)-H\left(U_{S \cap T} \mid Y_{S \cap T}, Q\right) \\
= & H\left(U_{S \backslash T} \mid U_{S^{c}}, Q\right)+H\left(U_{S \cap T} \mid U_{(S \cap T)^{c}}, Q\right)+H\left(U_{T} \mid U_{T^{c}}, Q\right) \\
& -H\left(U_{S \cup T} \mid Y_{S \cup T}, Q\right)-H\left(U_{S \cap T} \mid Y_{S \cap T}, Q\right) \\
= & H\left(U_{S \backslash T} \mid U_{S^{c}}, Q\right)+H\left(U_{T} \mid U_{T^{c}}, Q\right)-H\left(U_{S \cup T} \mid Y_{S \cup T}, Q\right) \\
& +I\left(U_{S \cap T} ; Y_{S \cap T} \mid U_{(S \cap T)^{c}}, Q\right) \\
\leq & H\left(U_{S \backslash T} \mid U_{(S \cup T)^{c}}, Q\right)+H\left(U_{T} \mid U_{T^{c}}, Q\right)-H\left(U_{S \cup T} \mid Y_{S \cup T}, Q\right) \\
& +I\left(U_{S \cap T} ; Y_{S \cap T} \mid U_{(S \cap T)^{c}}, Q\right) \\
= & I\left(U_{S \cup T} ; Y_{S \cup T} \mid U_{(S \cup T)^{c}}, Q\right)+I\left(U_{S \cap T} ; Y_{S \cap T} \mid U_{(S \cap T)^{c}}, Q\right) \\
= & f^{\prime}(S \cap T)+f^{\prime}(S \cup T) .
\end{aligned}
$$

The labeled steps above can be justified as follows:

- 37) follows since $U_{i}$ is conditionally independent of everything else given $\left(Y_{i}, Q\right)$.

- 38 is simply the chain rule.

- 39 follows since conditioning reduces entropy.

Next, we show that $f^{+}=\max \{f, 0\}$ is supermodular. Observe first that $f$ is monotone increasing, i.e., if $S \subset T$, then $f(S) \leq f(T)$. Thus, fixing $S, T \subseteq\{1, \ldots, m\}$, we can assume without loss of generality that

$$
f(S \cap T) \leq f(S) \leq f(T) \leq f(S \cup T) .
$$

If $f(S \cap T) \geq 0$, then (35) is satisfied for $s=f^{+}$by the supermodularity of $f$. On the other hand, if $f(S \cup T) \leq 0$, then (35) is a tautology for $s=f^{+}$. Therefore, it suffices to check the following three cases:

- Case 1: $f(S \cap T) \leq 0 \leq f(S) \leq f(T) \leq f(S \cup T)$. In this case, the supermodularity of $f$ and the fact that $f^{+} \geq f$ imply:

$$
\begin{aligned}
f^{+}(S \cup T)+f^{+}(S \cap T) & \geq f(S \cup T)+f(S \cap T) \\
& \geq f(S)+f(T)=f^{+}(S)+f^{+}(T) .
\end{aligned}
$$

- Case 2: $f(S \cap T) \leq f(S) \leq 0 \leq f(T) \leq f(S \cup T)$. Since $f$ is monotone increasing, we have:

$$
f^{+}(S \cup T)+f^{+}(S \cap T)=f(S \cup T)+0 \geq f(T)+0=f^{+}(S)+f^{+}(T) .
$$


- Case 3: $f(S \cap T) \leq f(S) \leq f(T) \leq 0 \leq f(S \cup T)$. By definition of $f^{+}$:

$$
f^{+}(S \cup T)+f^{+}(S \cap T)=f(S \cup T)+0 \geq 0+0=f^{+}(S)+f^{+}(T) .
$$

Hence, $f^{+}=\max \{f, 0\}$ is supermodular.

\section{Amplifying a Pointwise Convexity Constraint}

Lemma 7. Let $r_{1}, r_{2} \in \mathbb{R}$ be given, and suppose $f_{1}: K \rightarrow \mathbb{R}$ and $f_{2}: K \rightarrow \mathbb{R}$ are continuous functions defined on a compact domain $K \subset \mathbb{R}^{n}$. If there exists a function $h:[0,1] \rightarrow K$ satisfying

$$
t\left(f_{1} \circ h\right)(t)+(1-t)\left(f_{2} \circ h\right)(t) \leq t r_{1}+(1-t) r_{2} \text { for all } t \in[0,1],
$$

then there exists $x_{1}^{*}, x_{2}^{*} \in K$ and $t^{*} \in[0,1]$ for which

$$
\begin{aligned}
& t^{*} f_{1}\left(x_{1}^{*}\right)+\left(1-t^{*}\right) f_{1}\left(x_{2}^{*}\right) \leq r_{1} \\
& t^{*} f_{2}\left(x_{1}^{*}\right)+\left(1-t^{*}\right) f_{2}\left(x_{2}^{*}\right) \leq r_{2} .
\end{aligned}
$$

Before we prove the lemma, we make a few remarks. At first glance, this lemma appears somewhat bizarre. Indeed, the set $K$ need only be compact (e.g., connectedness is not required) and $h$ can be an arbitrarily complicated function, as long as it satisfies 40 . The strange nature of the lemma is echoed by the proof in that we merely prove the existence of the desired $x_{1}^{*}, x_{2}^{*}$ and $t^{*}$; no further information is obtained. Stripped to its core, the existence of the desired $x_{1}^{*}, x_{2}^{*}$ and $t^{*}$ essentially follows from the pigeonhole principle, which manifests itself in the sequential compactness of $K$.

Despite its strange nature, Lemma 7 is crucial in establishing the converse result for the multiterminal source coding problem under logarithmic loss. In this application, $K$ is taken to be a closed subset of a finite-dimensional probability simplex and $f_{1}, f_{2}$ are conditional entropies evaluated for probability distributions in $K$.

Finally, we remark that the Lemma 7 can be generalized to a certain extent. For example, the function $h$ need only be defined on a dense subset of $[0,1]$ and the set $K$ can be a more general sequentially compact space.

Proof of Lemma 7. Since $f_{1}, f_{2}$ are continuous and $K$ is compact, there exists $M<\infty$ such that $f_{1}$ and $f_{2}$ are bounded from above and below by $M$ and $-M$, respectively. Fix $\epsilon>0$, and partition the interval $[0,1]$ as $0=t_{1}<t_{2}<\cdots<$ $t_{m}=1$, such that $\left|t_{j+1}-t_{j}\right|<\frac{\epsilon}{M}$. For convenience define $x_{t_{j}}:=h\left(t_{j}\right)$ when $t_{j}$ is in the partition.

\footnotetext{
${ }^{4}$ Although not required for our purposes, we can assume $f_{1}$ and $f_{2}$ are defined and continuous over all of $\mathbb{R}^{n}$. This is a consequence of the Tietze extension theorem.
} 
Now, for $i=1,2$ define piecewise-linear functions $g_{1}(t), g_{2}(t)$ on $[0,1]$ by:

$$
g_{i}(t)= \begin{cases}f_{i}\left(x_{t_{j}}\right) & \text { if } t_{j} \text { is in the partition } \\ \theta f_{i}\left(x_{t_{j}}\right)+(1-\theta) f_{i}\left(x_{t_{j+1}}\right) & \text { if } t \text { is in the interval }\left(t_{j}, t_{j+1}\right),\end{cases}
$$

where $\theta \in(0,1)$ is chosen so that $t=\theta t_{j}+(1-\theta) t_{j+1}$ when $t$ is in the interval $\left(t_{j}, t_{j+1}\right)$.

With $g_{1}(t)$ and $g_{2}(t)$ defined in this manner, suppose $t=\theta t_{j}+(1-\theta) t_{j+1}$ for some $j$ and $\theta$. Then some straightforward algebra yields:

$$
\begin{aligned}
t g_{1}(t)+(1-t) g_{2}(t)= & \left(\theta t_{j}+(1-\theta) t_{j+1}\right)\left(\theta f_{1}\left(x_{t_{j}}\right)+(1-\theta) f_{1}\left(x_{t_{j+1}}\right)\right) \\
& +\left(1-\theta t_{j}-(1-\theta) t_{j+1}\right)\left(\theta f_{2}\left(x_{t_{j}}\right)+(1-\theta) f_{2}\left(x_{t_{j+1}}\right)\right) \\
= & \theta^{2}\left[t_{j} f_{1}\left(x_{t_{j}}\right)+\left(1-t_{j}\right) f_{2}\left(x_{t_{j}}\right)\right] \\
& +(1-\theta)^{2}\left[t_{j+1} f_{1}\left(x_{t_{j+1}}\right)+\left(1-t_{j+1}\right) f_{2}\left(x_{t_{j+1}}\right)\right] \\
& +\theta(1-\theta)\left[\left(1-t_{j}\right) f_{2}\left(x_{t_{j+1}}\right)+\left(1-t_{j+1}\right) f_{2}\left(x_{t_{j}}\right)\right. \\
& \left.+t_{j+1} f_{1}\left(x_{t_{j}}\right)+t_{j} f_{1}\left(x_{t_{j+1}}\right)\right] \\
\leq & \theta^{2}\left[t_{j} f_{1}\left(x_{t_{j}}\right)+\left(1-t_{j}\right) f_{2}\left(x_{t_{j}}\right)\right] \\
& +(1-\theta)^{2}\left[t_{j+1} f_{1}\left(x_{t_{j+1}}\right)+\left(1-t_{j+1}\right) f_{2}\left(x_{t_{j+1}}\right)\right] \\
& +\theta(1-\theta)\left[\left(1-t_{j+1}\right) f_{2}\left(x_{t_{j+1}}\right)+\left(1-t_{j}\right) f_{2}\left(x_{t_{j}}\right)\right. \\
& \left.\quad+t_{j} f_{1}\left(x_{t_{j}}\right)+t_{j+1} f_{1}\left(x_{t_{j+1}}\right)\right]+\epsilon \\
\leq & \theta^{2}\left[t_{j} r_{1}+\left(1-t_{j}\right) r_{2}\right] \\
& +(1-\theta)^{2}\left[t_{j+1} r_{1}+\left(1-t_{j+1}\right) r_{2}\right] \\
& +\theta(1-\theta)\left[\left(1-t_{j+1}\right) r_{2}+\left(1-t_{j}\right) r_{2}\right. \\
& \left.\quad+t_{j} r_{1}+t_{j+1} r_{1}\right]+\epsilon \\
= & \left(\theta t_{j}+(1-\theta) t_{j+1}\right) r_{1}+\left(1-\theta t_{j}-(1-\theta) t_{j+1}\right) r_{2}+\epsilon \\
= & t r_{1}+(1-t) r_{2}+\epsilon,
\end{aligned}
$$

where the first inequality follows since $\left|t_{j+1}-t_{j}\right|$ is small, and the second inequality follows from the the fact that 400 holds for each $t_{j}$ in the partition. Notably, this implies that it is impossible to have

$$
g_{1}(t)>r_{1}+\epsilon \text { and } g_{2}(t)>r_{2}+\epsilon
$$

hold simultaneously for any $t \in[0,1]$, else we would obtain a contradiction to (42). Also, since we included the endpoints $t_{1}=0$ and $t_{m}=1$ in the partition, we have the following two inequalities:

$$
g_{1}(1) \leq r_{1} \text {, and } g_{2}(0) \leq r_{2} .
$$

Combining these observations with the fact that $g_{1}(t)$ and $g_{2}(t)$ are continuous, there must exist some $t^{*} \in[0,1]$ for which

$$
g_{1}\left(t^{*}\right) \leq r_{1}+\epsilon, \text { and } g_{2}\left(t^{*}\right) \leq r_{2}+\epsilon
$$




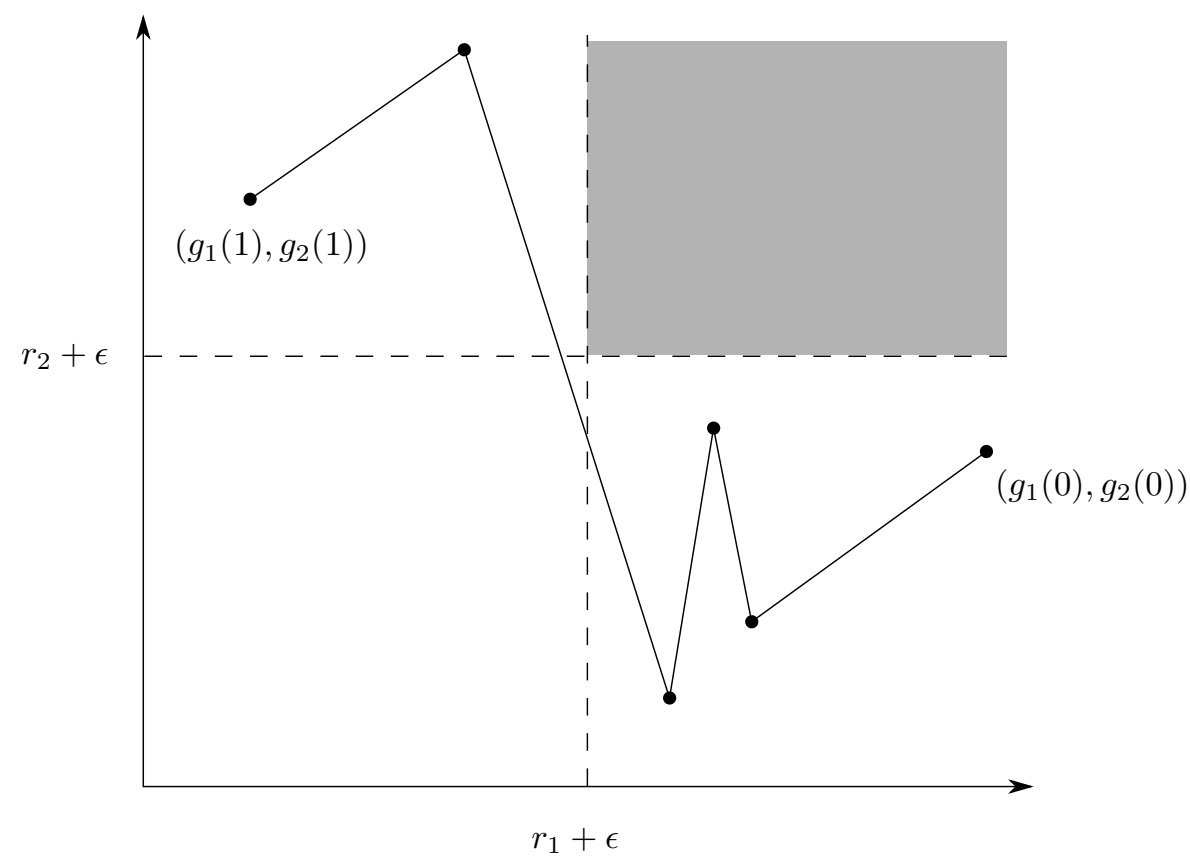

Figure 5: A parametric plot of the function $\varphi: t \mapsto\left(g_{1}(t), g_{2}(t)\right)$. Since $\varphi(t)$ is continuous, starts with $g_{2}(0) \leq r_{2}+\epsilon$, ends with $g_{1}(1) \leq r_{1}+\epsilon$, and doesn't intersect the shaded area, $\varphi(t)$ must pass through the lower-left region.

simultaneously. An illustration of this is given in Figure 5 which is a mere variation on the classical intermediate value theorem.

Applying this result, we can find a sequence $\left\{x_{1}^{(n)}, x_{2}^{(n)}, t^{(n)}\right\}_{n=1}^{\infty}$ satisfying

$$
\begin{aligned}
t^{(n)} f_{1}\left(x_{1}^{(n)}\right)+\left(1-t^{(n)}\right) f_{1}\left(x_{2}^{(n)}\right) & \leq r_{1}+\frac{1}{n} \\
t^{(n)} f_{2}\left(x_{1}^{(n)}\right)+\left(1-t^{(n)}\right) f_{2}\left(x_{2}^{(n)}\right) & \leq r_{2}+\frac{1}{n}
\end{aligned}
$$

for each $n \geq 1$. Since $K \times K \times[0,1]$ is sequentially compact, there exists a convergent subsequence $\left\{n_{j}\right\}_{j=1}^{\infty}$ such that $\left(x_{1}^{\left(n_{j}\right)}, x_{2}^{\left(n_{j}\right)}, t^{\left(n_{j}\right)}\right) \rightarrow\left(x_{1}^{*}, x_{2}^{*}, t^{*}\right) \in$ $K \times K \times[0,1]$. The continuity of $f_{1}$ and $f_{2}$ then apply to yield the desired result.

\section{E Strengthening the Converse of Theorem 6}

In this appendix, we prove a stronger version of the converse of Theorem 6 To be precise, let $\hat{\mathcal{Y}}_{1}^{* n}$ and $\hat{\mathcal{Y}}_{2}^{* n}$ denote the set of probability measures on $\mathcal{Y}_{1}^{n}$ and $\mathcal{Y}_{2}^{n}$, respectively. Let $d_{1}^{*}, d_{2}^{*}$ be the (extended)-log loss distortion measures 
defined as follows:

$$
\begin{aligned}
& d_{1}^{*}\left(y_{1}^{n}, \hat{y}_{1}^{n}\right)=\frac{1}{n} \log \left(\frac{1}{\hat{y}_{1}^{n}\left(y_{1}^{n}\right)}\right) \\
& d_{2}^{*}\left(y_{2}^{n}, \hat{y}_{2}^{n}\right)=\frac{1}{n} \log \left(\frac{1}{\hat{y}_{2}^{n}\left(y_{2}^{n}\right)}\right),
\end{aligned}
$$

where $\hat{y}_{1}^{n}\left(y_{1}^{n}\right)$ is the probability assigned to outcome $y_{1}^{n} \in \mathcal{Y}_{1}^{n}$ by the probability measure $\hat{y}_{1}^{n} \in \hat{\mathcal{Y}}_{1}^{* n}$. Similarly for $\hat{y}_{2}^{n}\left(y_{2}^{n}\right)$. Note that this extends the standard definition of logarithmic loss to sequence reproductions.

Definition 10. We say that a tuple $\left(R_{1}, R_{2}, D_{1}, D_{2}\right)$ is sequence-achievable if, for any $\epsilon>0$, there exist encoding functions

$$
\begin{aligned}
& f_{1}: \mathcal{Y}_{1}^{n} \rightarrow\left\{1, \ldots, 2^{n R_{1}}\right\} \\
& f_{2}: \mathcal{Y}_{2}^{n} \rightarrow\left\{1, \ldots, 2^{n R_{2}}\right\},
\end{aligned}
$$

and decoding functions

$$
\begin{aligned}
& \phi_{1}:\left\{1, \ldots, 2^{n R_{1}}\right\} \times\left\{1, \ldots, 2^{n R_{2}}\right\} \rightarrow \hat{\mathcal{Y}}_{1}^{* n} \\
& \phi_{2}:\left\{1, \ldots, 2^{n R_{1}}\right\} \times\left\{1, \ldots, 2^{n R_{2}}\right\} \rightarrow \hat{\mathcal{Y}}_{2}^{* n}
\end{aligned}
$$

which satisfy

$$
\begin{aligned}
& \mathbb{E} d_{1}^{*}\left(Y_{1}^{n}, \hat{Y}_{1}^{n}\right) \leq D_{1}+\epsilon \\
& \mathbb{E} d_{2}^{*}\left(Y_{2}^{n}, \hat{Y}_{2}^{n}\right) \leq D_{2}+\epsilon
\end{aligned}
$$

where

$$
\begin{aligned}
& \hat{Y}_{1}^{n}=\phi_{1}\left(f_{1}\left(Y_{1}^{n}\right), f_{2}\left(Y_{2}^{n}\right)\right) \\
& \hat{Y}_{2}^{n}=\phi_{2}\left(f_{1}\left(Y_{1}^{n}\right), f_{2}\left(Y_{2}^{n}\right)\right) .
\end{aligned}
$$

Theorem 13. If $\left(R_{1}, R_{2}, D_{1}, D_{2}\right)$ is sequence-achievable, then $\left(R_{1}, R_{2}, D_{1}, D_{2}\right) \in \mathcal{R} \mathcal{D}^{i}=\overline{\mathcal{R D}}^{\star}$.

Proof. The theorem is an immediate consequence of Theorem 6 and Lemmas 8 and 9, which are given below.

Remark 4. We refer to Theorem 13 as the "strengthened converse" of Theorem 6. Indeed, it states that enlarging the set of possible reproduction sequences to include non-product distributions cannot attain better performance than when the decoder is restricted to choosing a reproduction sequence from the set of product distributions.

Lemma 8. If $\left(R_{1}, R_{2}, \tilde{D}_{1}, D_{2}\right)$ is sequence-achievable, then there exists a joint distribution

$$
p\left(y_{1}, y_{2}, u_{1}, u_{2}, q\right)=p(q) p\left(y_{1}, y_{2}\right) p\left(u_{1} \mid y_{1}, q\right) p\left(u_{2} \mid y_{2}, q\right)
$$


and a $D_{1} \leq \tilde{D}_{1}$ which satisfies

$$
\begin{aligned}
& D_{1} \geq H\left(Y_{1} \mid U_{1}, U_{2}, Q\right) \\
& D_{2} \geq D_{1}+H\left(Y_{2} \mid U_{1}, U_{2}, Q\right)-H\left(Y_{1} \mid U_{1}, U_{2}, Q\right),
\end{aligned}
$$

and

$$
\begin{aligned}
R_{1} & \geq H\left(Y_{1} \mid U_{2}, Q\right)-D_{1} \\
R_{2} & \geq I\left(Y_{2} ; U_{2} \mid Y_{1}, Q\right)+H\left(Y_{1} \mid U_{1}, Q\right)-D_{1} \\
R_{1}+R_{2} & \geq I\left(Y_{2} ; U_{2} \mid Y_{1}, Q\right)+H\left(Y_{1}\right)-D_{1} .
\end{aligned}
$$

Proof. For convenience, let $F_{1}=f_{1}\left(Y_{1}^{n}\right)$ and $F_{2}=f_{2}\left(Y_{2}^{n}\right)$, where $f_{1}, f_{2}$ are the encoding functions corresponding to a scheme which achieves $\left(R_{1}, R_{2}, \tilde{D}_{1}, D_{2}\right)$ (in the sequence-reproduction sense). Define $D_{1}=\frac{1}{n} H\left(Y_{1}^{n} \mid F_{1}, F_{2}\right)$, so that:

$$
n D_{1}=H\left(Y_{1}^{n} \mid F_{1}, F_{2}\right) .
$$

Since $n \tilde{D}_{1} \geq H\left(Y_{1}^{n} \mid F_{1}, F_{2}\right)$ by the strengthened version ${ }^{5}$ of Lemma 1 we have $D_{1} \leq \tilde{D}_{1}$ as desired. By definition of $D_{1}$, we immediately obtain the following inequality:

$$
n D_{1}=\sum_{i=1}^{n} H\left(Y_{1, i} \mid F_{1}, F_{2}, Y_{1, i+1}^{n}\right) \geq \sum_{i=1}^{n} H\left(Y_{1, i} \mid F_{1}, F_{2}, Y_{2}^{i-1}, Y_{1, i+1}^{n}\right) .
$$

Next, recall the Csiszár sum identity:

$$
\sum_{i=1}^{n} I\left(Y_{1, i+1}^{n} ; Y_{2, i} \mid Y_{2}^{i-1}, F_{1}, F_{2}\right)=\sum_{i=1}^{n} I\left(Y_{2}^{i-1} ; Y_{1, i} \mid Y_{1, i+1}^{n}, F_{1}, F_{2}\right) .
$$

This, together with (43), implies the following inequality:

$$
n D_{2} \geq n D_{1}+\sum_{i=1}^{n} H\left(Y_{2, i} \mid F_{1}, F_{2}, Y_{2}^{i-1}, Y_{1, i+1}^{n}\right)-H\left(Y_{1, i} \mid F_{1}, F_{2}, Y_{2}^{i-1}, Y_{1, i+1}^{n}\right),
$$

\footnotetext{
${ }^{5}$ See the comment in Section 3.3
} 
which we can verifiy as follows:

$$
\begin{aligned}
n D_{2} & \geq H\left(Y_{2}^{n} \mid F_{1}, F_{2}\right)=\sum_{i=1}^{n} H\left(Y_{2, i} \mid F_{1}, F_{2}, Y_{2}^{i-1}\right) \\
& =\sum_{i=1}^{n} H\left(Y_{2, i} \mid F_{1}, F_{2}, Y_{2}^{i-1}, Y_{1, i+1}^{n}\right)+I\left(Y_{1, i+1}^{n} ; Y_{2, i} \mid F_{1}, F_{2}, Y_{2}^{i-1}\right) \\
& =\sum_{i=1}^{n} H\left(Y_{2, i} \mid F_{1}, F_{2}, Y_{2}^{i-1}, Y_{1, i+1}^{n}\right)+I\left(Y_{2}^{i-1} ; Y_{1, i} \mid Y_{1, i+1}^{n}, F_{1}, F_{2}\right) \\
& =H\left(Y_{1}^{n} \mid F_{1}, F_{2}\right)+\sum_{i=1}^{n} H\left(Y_{2, i} \mid F_{1}, F_{2}, Y_{2}^{i-1}, Y_{1, i+1}^{n}\right)-H\left(Y_{1, i} \mid F_{1}, F_{2}, Y_{2}^{i-1}, Y_{1, i+1}^{n}\right) \\
& =n D_{1}+\sum_{i=1}^{n} H\left(Y_{2, i} \mid F_{1}, F_{2}, Y_{2}^{i-1}, Y_{1, i+1}^{n}\right)-H\left(Y_{1, i} \mid F_{1}, F_{2}, Y_{2}^{i-1}, Y_{1, i+1}^{n}\right) .
\end{aligned}
$$

Next, observe that we can lower bound $R_{1}$ as follows:

$$
\begin{aligned}
n R_{1} & \geq H\left(F_{1}\right) \geq I\left(Y_{1}^{n} ; F_{1} \mid F_{2}\right) \\
& =\sum_{i=1}^{n} H\left(Y_{1, i} \mid F_{2}, Y_{1}^{i-1}\right)-H\left(Y_{1}^{n} \mid F_{1}, F_{2}\right) \\
& \geq \sum_{i=1}^{n} H\left(Y_{1, i} \mid F_{2}, Y_{1}^{i-1}, Y_{2}^{i-1}\right)-n D_{1} \\
& =\sum_{i=1}^{n} H\left(Y_{1, i} \mid F_{2}, Y_{2}^{i-1}\right)-n D_{1} \\
& \geq \sum_{i=1}^{n} H\left(Y_{1, i} \mid F_{2}, Y_{2}^{i-1}, Y_{1, i+1}^{n}\right)-n D_{1} .
\end{aligned}
$$

In the above string of inequalities, 46 follows from 430 and the fact that conditioning reduces entropy. Equality 477 follows since $Y_{1, i} \leftrightarrow F_{2}, Y_{2}^{i-1} \leftrightarrow$ $Y_{1}^{i-1}$ form a Markov chain (in that order). 
Next, we can obtain a lower bound on $R_{2}$ :

$$
\begin{aligned}
n R_{2} & \geq H\left(F_{2}\right) \geq H\left(F_{2} \mid F_{1}\right)=H\left(F_{2} \mid F_{1}, Y_{1}^{n}\right)+I\left(Y_{1}^{n} ; F_{2} \mid F_{1}\right) \\
& \geq I\left(Y_{2}^{n} ; F_{2} \mid F_{1}, Y_{1}^{n}\right)+I\left(Y_{1}^{n} ; F_{2} \mid F_{1}\right) \\
& =I\left(Y_{2}^{n} ; F_{2} \mid Y_{1}^{n}\right)+I\left(Y_{1}^{n} ; F_{2} \mid F_{1}\right) \\
& =\sum_{i=1}^{n} I\left(Y_{2, i} ; F_{2} \mid Y_{1}^{n}, Y_{2}^{i-1}\right)+H\left(Y_{1, i} \mid F_{1}, Y_{1, i+1}^{n}\right)-n D_{1} \\
& \geq \sum_{i=1}^{n} I\left(Y_{2, i} ; F_{2} \mid Y_{1}^{n}, Y_{2}^{i-1}\right)+H\left(Y_{1, i} \mid F_{1}, Y_{2}^{i-1}, Y_{1, i+1}^{n}\right)-n D_{1} \\
& =\sum_{i=1}^{n} I\left(Y_{2, i} ; F_{2}, Y_{1}^{i-1}, Y_{2}^{i-1} \mid Y_{1, i}, Y_{2}^{i-1}, Y_{1, i+1}^{n}\right)+H\left(Y_{1, i} \mid F_{1}, Y_{2}^{i-1}, Y_{1, i+1}^{n}\right)-n D_{1} \\
& \geq \sum_{i=1}^{n} I\left(Y_{2, i} ; F_{2}, Y_{2}^{i-1} \mid Y_{1, i}, Y_{2}^{i-1}, Y_{1, i+1}^{n}\right)+H\left(Y_{1, i} \mid F_{1}, Y_{2}^{i-1}, Y_{1, i+1}^{n}\right)-n D_{1} .
\end{aligned}
$$

In the above string of inequalities, (50) follows from (43) and the chain rule. (51) follows from the i.i.d. property of the sources, and $(52)$ follows by monotonicity of mutual information.

A lower bound on the sum-rate $R_{1}+R_{2}$ can be obtained as follows:

$$
\begin{aligned}
n\left(R_{1}+R_{2}\right) & \geq H\left(F_{1}\right)+H\left(F_{2}\right) \geq H\left(F_{2}\right)+H\left(F_{1} \mid F_{2}\right) \\
& \geq I\left(F_{2} ; Y_{1}^{n}, Y_{2}^{n}\right)+I\left(F_{1} ; Y_{1}^{n} \mid F_{2}\right) \\
& =I\left(F_{2} ; Y_{1}^{n}\right)+I\left(F_{2} ; Y_{2}^{n} \mid Y_{1}^{n}\right)+I\left(F_{1} ; Y_{1}^{n} \mid F_{2}\right) \\
& =I\left(F_{2} ; Y_{2}^{n} \mid Y_{1}^{n}\right)+I\left(F_{1}, F_{2} ; Y_{1}^{n}\right) \\
& \geq \sum_{i=1}^{n} I\left(Y_{2, i} ; F_{2}, Y_{2}^{i-1} \mid Y_{1, i}, Y_{2}^{i-1}, Y_{1, i+1}^{n}\right)+H\left(Y_{1, i}\right)-n D_{1} .
\end{aligned}
$$

Where (53) follows in a manner similar to 490 - 52 in the lower bound on $R_{2}$.

Now, define $U_{1, i} \triangleq F_{1}, U_{2, i} \triangleq\left(F_{2}, Y_{2}^{i-1}\right)$, and $Q_{i} \triangleq\left(Y_{2}^{i-1}, Y_{1, i+1}^{n}\right)$. Then we can summarize our results so far as follows. Inequalities (44) and 45) become

$$
\begin{aligned}
& D_{1} \geq \frac{1}{n} \sum_{i=1}^{n} H\left(Y_{1, i} \mid U_{1, i}, U_{2, i}, Q_{i}\right) \\
& D_{2} \geq D_{1}+\frac{1}{n} \sum_{i=1}^{n} H\left(Y_{2, i} \mid U_{1, i}, U_{2, i}, Q_{i}\right)-H\left(Y_{1, i} \mid U_{1, i}, U_{2, i}, Q_{i}\right),
\end{aligned}
$$


and inequalities (48), (52), and (53) can be written as:

$$
\begin{aligned}
R_{1} & \geq \frac{1}{n} \sum_{i=1}^{n} H\left(Y_{1, i} \mid U_{2, i}, Q_{i}\right)-D_{1} \\
R_{2} & \geq \frac{1}{n} \sum_{i=1}^{n} I\left(Y_{2, i} ; U_{2, i} \mid Y_{1, i}, Q_{i}\right)+H\left(Y_{1, i} \mid U_{1, i}, Q_{i}\right)-D_{1} \\
R_{1}+R_{2} & \geq \frac{1}{n} \sum_{i=1}^{n} I\left(Y_{2, i} ; U_{2, i} \mid Y_{1, i}, Q_{i}\right)+H\left(Y_{1, i}\right)-D_{1} .
\end{aligned}
$$

Next, we note that $U_{1, i} \leftrightarrow Y_{1, i} \leftrightarrow Y_{2, i} \leftrightarrow U_{2, i}$ form a Markov chain (in that order) conditioned on $Q_{i}$. Moreover, $Q_{i}$ is independent of $Y_{1, i}, Y_{2, i}$. Hence, a standard timesharing argument proves the lemma.

Lemma 9. Fix $\left(R_{1}, R_{2}, D_{1}, D_{2}\right)$. If there exists a joint distribution of the form

$$
p\left(y_{1}, y_{2}, u_{1}, u_{2}, q\right)=p(q) p\left(y_{1}, y_{2}\right) p\left(u_{1} \mid y_{1}, q\right) p\left(u_{2} \mid y_{2}, q\right)
$$

which satisfies

$$
\begin{aligned}
& D_{1} \geq H\left(Y_{1} \mid U_{1}, U_{2}, Q\right) \\
& D_{2} \geq D_{1}+H\left(Y_{2} \mid U_{1}, U_{2}, Q\right)-H\left(Y_{1} \mid U_{1}, U_{2}, Q\right),
\end{aligned}
$$

and

$$
\begin{aligned}
R_{1} & \geq H\left(Y_{1} \mid U_{2}, Q\right)-D_{1} \\
R_{2} & \geq I\left(Y_{2} ; U_{2} \mid Y_{1}, Q\right)+H\left(Y_{1} \mid U_{1}, Q\right)-D_{1} \\
R_{1}+R_{2} & \geq I\left(Y_{2} ; U_{2} \mid Y_{1}, Q\right)+H\left(Y_{1}\right)-D_{1},
\end{aligned}
$$

then $\left(R_{1}, R_{2}, D_{1}, D_{2}\right) \in \mathcal{R} \mathcal{D}^{i}$.

Proof. Let $\mathcal{P}$ denote the polytope of rate pairs which satisfy the inequalities (56)-(58). It suffices to show that if $\left(r_{1}, r_{2}\right)$ is a vertex of $\mathcal{P}$, then $\left(r_{1}, r_{2}, D_{1}, D_{2}\right) \in \mathcal{R D}^{i}$. For convenience, let $[x]^{+}=\max \{x, 0\}$. There are only two extreme points of $\mathcal{P}$ :

$$
\begin{aligned}
& r_{1}^{(1)}=\left[H\left(Y_{1} \mid U_{2}, Q\right)-D_{1}\right]^{+} \\
& r_{2}^{(1)}=I\left(Y_{2} ; U_{2} \mid Y_{1}, Q\right)+H\left(Y_{1}\right)-D_{1}-r_{1}^{(1)},
\end{aligned}
$$

and

$$
\begin{aligned}
& r_{1}^{(2)}=I\left(Y_{2} ; U_{2} \mid Y_{1}, Q\right)+H\left(Y_{1}\right)-D_{1}-r_{2}^{(2)}, \\
& r_{2}^{(2)}=\left[I\left(Y_{2} ; U_{2} \mid Y_{1}, Q\right)+H\left(Y_{1} \mid U_{1}, Q\right)-D_{1}\right]^{+} .
\end{aligned}
$$

We first analyze the extreme point $\left(r_{1}^{(1)}, r_{2}^{(1)}\right)$ : 
- Case 1.1: $r_{1}^{(1)}=0$. In this case, we have $r_{2}^{(1)}=I\left(Y_{2} ; U_{2} \mid Y_{1}, Q\right)+H\left(Y_{1}\right)-$ $D_{1}$. This can be expressed as:

$$
r_{2}^{(1)}=(1-\theta) I\left(Y_{2} ; U_{2} \mid Q\right),
$$

where

$$
\theta=\frac{D_{1}-I\left(Y_{2} ; U_{2} \mid Y_{1}, Q\right)-H\left(Y_{1}\right)+I\left(Y_{2} ; U_{2} \mid Q\right)}{I\left(Y_{2} ; U_{2} \mid Q\right)} .
$$

Since $r_{1}^{(1)}=0$, we must have $D_{1} \geq H\left(Y_{1} \mid U_{2}, Q\right)$. This implies that

$$
\theta \geq \frac{H\left(Y_{1} \mid U_{2}, Q\right)-I\left(Y_{2} ; U_{2} \mid Y_{1}, Q\right)-H\left(Y_{1}\right)+I\left(Y_{2} ; U_{2} \mid Q\right)}{I\left(Y_{2} ; U_{2} \mid Q\right)}=0 .
$$

Also, we can assume without loss of generality that $D_{1} \leq H\left(Y_{1}\right)$, hence $\theta \in[0,1]$. Applying the Berger-Tung achievability scheme, we can achieve the following distortions:

$$
\begin{aligned}
D_{1}^{\theta} & =\theta H\left(Y_{1}\right)+(1-\theta) H\left(Y_{1} \mid U_{2}, Q\right) \\
& =H\left(Y_{1} \mid U_{2}, Q\right)+\theta I\left(Y_{1} ; U_{2} \mid Q\right) \\
& \leq H\left(Y_{1} \mid U_{2}, Q\right)+D_{1}-I\left(Y_{2} ; U_{2} \mid Y_{1}, Q\right)-H\left(Y_{1}\right)+I\left(Y_{2} ; U_{2} \mid Q\right) \\
& =D_{1}-I\left(Y_{2} ; U_{2} \mid Y_{1}, Q\right)-I\left(Y_{1} ; U_{2} \mid Q\right)+I\left(Y_{2} ; U_{2} \mid Q\right) \\
& =D_{1},
\end{aligned}
$$

where 59 follows since $I\left(Y_{1} ; U_{2} \mid Q\right) \leq I\left(Y_{2} ; U_{2} \mid Q\right)$ by the data processing inequality.

$$
\begin{aligned}
D_{2}^{\theta} & =\theta H\left(Y_{2}\right)+(1-\theta) H\left(Y_{2} \mid U_{2}, Q\right) \\
& =H\left(Y_{2} \mid U_{2}, Q\right)+\theta I\left(Y_{2} ; U_{2} \mid Q\right) \\
& =H\left(Y_{2} \mid U_{2}, Q\right)+D_{1}-I\left(Y_{2} ; U_{2} \mid Y_{1}, Q\right)-H\left(Y_{1}\right)+I\left(Y_{2} ; U_{2} \mid Q\right) \\
& =H\left(Y_{2}\right)+D_{1}-I\left(Y_{2} ; U_{2} \mid Y_{1}, Q\right)-H\left(Y_{1}\right) \\
& =H\left(Y_{2} \mid Y_{1}, U_{2}, Q\right)+D_{1}-H\left(Y_{1} \mid Y_{2}\right) \\
& =H\left(Y_{2} \mid Y_{1}, U_{1}, U_{2}, Q\right)+D_{1}-H\left(Y_{1} \mid Y_{2}\right) \\
& \leq H\left(Y_{2} \mid Y_{1}, U_{1}, U_{2}, Q\right)+D_{1}-H\left(Y_{1} \mid Y_{2}, U_{1}, U_{2}, Q\right) \\
& =H\left(Y_{2} \mid U_{1}, U_{2}, Q\right)+D_{1}-H\left(Y_{1} \mid U_{1}, U_{2}, Q\right) \\
& \leq D_{2},
\end{aligned}
$$

where 60 follows since $U_{1} \leftrightarrow\left(Y_{1}, U_{2}, Q\right) \leftrightarrow Y_{2}$, and 61 follows from (55).

- Case 1.2: $r_{1}^{(1)} \geq 0$. In this case, we have $r_{2}^{(1)}=I\left(Y_{2} ; U_{2} \mid Y_{1}, Q\right)+$ $I\left(Y_{1} ; U_{2} \mid Q\right)=I\left(Y_{2} ; U_{2} \mid Q\right)$. Also, we can write $r_{1}^{(1)}$ as:

$$
r_{1}^{(1)}=(1-\theta) I\left(Y_{1} ; U_{1} \mid U_{2}, Q\right),
$$


where

$$
\theta=\frac{D_{1}-H\left(Y_{1} \mid U_{2}, Q\right)+I\left(Y_{1} ; U_{1} \mid U_{2}, Q\right)}{I\left(Y_{1} ; U_{1} \mid U_{2}, Q\right)} .
$$

Since $r_{1}^{(1)} \geq 0$, we must have $D_{1} \leq H\left(Y_{1} \mid U_{2}, Q\right)$. This implies that

$$
\theta \leq \frac{H\left(Y_{1} \mid U_{2}, Q\right)-H\left(Y_{1} \mid U_{2}, Q\right)+I\left(Y_{1} ; U_{1} \mid U_{2}, Q\right)}{I\left(Y_{1} ; U_{1} \mid U_{2}, Q\right)}=1 .
$$

Also, (54) implies that $D_{1} \geq H\left(Y_{1} \mid U_{1}, U_{2}, Q\right)$, hence $\theta \in[0,1]$. Applying the Berger-Tung achievability scheme, we can achieve the following distortions:

$$
\begin{aligned}
D_{1}^{\theta} & =\theta H\left(Y_{1} \mid U_{2}, Q\right)+(1-\theta) H\left(Y_{1} \mid U_{1}, U_{2}, Q\right) \\
& =H\left(Y_{1} \mid U_{1}, U_{2}, Q\right)+\theta I\left(Y_{1} ; U_{1} \mid U_{2}, Q\right) \\
& =H\left(Y_{1} \mid U_{1}, U_{2}, Q\right)+D_{1}-H\left(Y_{1} \mid U_{2}, Q\right)+I\left(Y_{1} ; U_{1} \mid U_{2}, Q\right) \\
& =D_{1},
\end{aligned}
$$

and

$$
\begin{aligned}
D_{2}^{\theta} & =\theta H\left(Y_{2} \mid U_{2}, Q\right)+(1-\theta) H\left(Y_{2} \mid U_{1}, U_{2}, Q\right) \\
& =H\left(Y_{2} \mid U_{1}, U_{2}, Q\right)+\theta I\left(Y_{2} ; U_{1} \mid U_{2}, Q\right) \\
& \leq H\left(Y_{2} \mid U_{1}, U_{2}, Q\right)+D_{1}-H\left(Y_{1} \mid U_{2}, Q\right)+I\left(Y_{1} ; U_{1} \mid U_{2}, Q\right) \\
& =H\left(Y_{2} \mid U_{1}, U_{2}, Q\right)+D_{1}-H\left(Y_{1} \mid U_{1}, U_{2}, Q\right) \\
& \leq D_{2},
\end{aligned}
$$

where 62 follows since $I\left(Y_{2} ; U_{1} \mid U_{2}, Q\right) \leq I\left(Y_{1} ; U_{1} \mid U_{2}, Q\right)$ by the data processing inequality, and 63 follows from 55.

In a similar manner, we now analyze the second extreme point $\left(r_{1}^{(2)}, r_{2}^{(2)}\right)$ :

- Case 2.1: $r_{2}^{(2)}=0$. In this case, we have $r_{1}^{(2)}=I\left(Y_{2} ; U_{2} \mid Y_{1}, Q\right)+H\left(Y_{1}\right)-$ $D_{1}$. This can be expressed as:

$$
r_{1}^{(2)}=(1-\theta) I\left(Y_{1} ; U_{1} \mid Q\right),
$$

where

$$
\theta=\frac{D_{1}-I\left(Y_{2} ; U_{2} \mid Y_{1}, Q\right)-H\left(Y_{1}\right)+I\left(Y_{1} ; U_{1} \mid Q\right)}{I\left(Y_{1} ; U_{1} \mid Q\right)} .
$$

Since $r_{2}^{(2)}=0$, we must have $D_{1} \geq H\left(Y_{1} \mid U_{1}, Q\right)+I\left(Y_{2} ; U_{2} \mid Y_{1}, Q\right)$. This implies that

$$
\theta \geq \frac{H\left(Y_{1} \mid U_{1}, Q\right)+I\left(Y_{2} ; U_{2} \mid Y_{1}, Q\right)-I\left(Y_{2} ; U_{2} \mid Y_{1}, Q\right)-H\left(Y_{1}\right)+I\left(Y_{1} ; U_{1} \mid Q\right)}{I\left(Y_{1} ; U_{1} \mid Q\right)}=0 .
$$


Also, we can assume without loss of generality that $D_{1} \leq H\left(Y_{1}\right)$, hence

$$
\theta \leq \frac{H\left(Y_{1}\right)-I\left(Y_{2} ; U_{2} \mid Y_{1}, Q\right)-H\left(Y_{1}\right)+I\left(Y_{1} ; U_{1} \mid Q\right)}{I\left(Y_{1} ; U_{1} \mid Q\right)} \leq 1
$$

and therefore $\theta \in[0,1]$. Applying the Berger-Tung achievability scheme, we can achieve the following distortions:

$$
\begin{aligned}
D_{1}^{\theta} & =\theta H\left(Y_{1}\right)+(1-\theta) H\left(Y_{1} \mid U_{1}, Q\right) \\
& =H\left(Y_{1} \mid U_{1}, Q\right)+\theta I\left(Y_{1} ; U_{1} \mid Q\right) \\
& =H\left(Y_{1} \mid U_{1}, Q\right)+D_{1}-I\left(Y_{2} ; U_{2} \mid Y_{1}, Q\right)-H\left(Y_{1}\right)+I\left(Y_{1} ; U_{1} \mid Q\right) \\
& =D_{1}-I\left(Y_{2} ; U_{2} \mid Y_{1}, Q\right) \\
& \leq D_{1}
\end{aligned}
$$

and

$$
\begin{aligned}
D_{2}^{\theta} & =\theta H\left(Y_{2}\right)+(1-\theta) H\left(Y_{2} \mid U_{1}, Q\right) \\
& =H\left(Y_{2} \mid U_{1}, Q\right)+\theta I\left(Y_{2} ; U_{1} \mid Q\right) \\
& \leq H\left(Y_{2} \mid U_{1}, Q\right)+D_{1}-I\left(Y_{2} ; U_{2} \mid Y_{1}, Q\right)-H\left(Y_{1}\right)+I\left(Y_{1} ; U_{1} \mid Q\right) \\
& =H\left(Y_{2} \mid Y_{1}, U_{2}, Q\right)+D_{1}-H\left(Y_{1} \mid Y_{2}, U_{1}, Q\right) \\
& =H\left(Y_{2} \mid Y_{1}, U_{1}, U_{2}, Q\right)+D_{1}-H\left(Y_{1} \mid Y_{2}, U_{1}, U_{2}, Q\right) \\
& =H\left(Y_{2} \mid U_{1}, U_{2}, Q\right)+D_{1}-H\left(Y_{1} \mid U_{1}, U_{2}, Q\right) \\
& \leq D_{2},
\end{aligned}
$$

where 64 follows since $I\left(Y_{2} ; U_{1} \mid Q\right) \leq I\left(Y_{1} ; U_{1} \mid Q\right)$ by the data processing inequality, 65 follows since $U_{1} \leftrightarrow\left(Y_{1}, U_{2}, Q\right) \leftrightarrow Y_{2}$ and $U_{2} \leftrightarrow$ $\left(Y_{2}, U_{1}, Q\right) \leftrightarrow Y_{1}$, and (66) follows from (55).

- Case 2.2: $r_{2}^{(2)} \geq 0$. In this case, we have $r_{1}^{(2)}=I\left(Y_{1} ; U_{1} \mid Q\right)$. Also, we can write $r_{2}^{(2)}$ as:

$$
r_{2}^{(2)}=(1-\theta) I\left(Y_{2} ; U_{2} \mid U_{1}, Q\right),
$$

where

$$
\theta=\frac{D_{1}-H\left(Y_{1} \mid U_{1}, Q\right)-I\left(Y_{2} ; U_{2} \mid Y_{1}, Q\right)+I\left(Y_{2} ; U_{2} \mid U_{1}, Q\right)}{I\left(Y_{2} ; U_{2} \mid U_{1}, Q\right)}
$$

Since $r_{2}^{(2)} \geq 0$, we must have $D_{1} \leq H\left(Y_{1} \mid U_{1}, Q\right)+I\left(Y_{2} ; U_{2} \mid Y_{1}, Q\right)$. This implies that $\theta \leq 1$. Also, (54) implies that $D_{1} \geq H\left(Y_{1} \mid U_{1}, U_{2}, Q\right)$, yielding $\theta \geq \frac{H\left(Y_{1} \mid U_{1}, U_{2}, Q\right)-H\left(Y_{1} \mid U_{1}, Q\right)-I\left(Y_{2} ; U_{2} \mid Y_{1}, Q\right)+I\left(Y_{2} ; U_{2} \mid U_{1}, Q\right)}{I\left(Y_{2} ; U_{2} \mid U_{1}, Q\right)}=0$. 
Therefore, $\theta \in[0,1]$. Applying the Berger-Tung achievability scheme, we can achieve the following distortions:

$$
\begin{aligned}
D_{1}^{\theta} & =\theta H\left(Y_{1} \mid U_{1}, Q\right)+(1-\theta) H\left(Y_{1} \mid U_{1}, U_{2}, Q\right) \\
& =H\left(Y_{1} \mid U_{1}, U_{2}, Q\right)+\theta I\left(Y_{1} ; U_{2} \mid U_{1}, Q\right) \\
& \leq H\left(Y_{1} \mid U_{1}, U_{2}, Q\right)+D_{1}-H\left(Y_{1} \mid U_{1}, Q\right)-I\left(Y_{2} ; U_{2} \mid Y_{1}, Q\right)+I\left(Y_{2} ; U_{2} \mid U_{1}, Q\right)
\end{aligned}
$$

$=D_{1}$,

where (67) follows since $I\left(Y_{1} ; U_{2} \mid U_{1}, Q\right) \leq I\left(Y_{2} ; U_{2} \mid U_{1}, Q\right)$ by the data processing inequality.

$$
\begin{aligned}
D_{2}^{\theta} & =\theta H\left(Y_{2} \mid U_{1}, Q\right)+(1-\theta) H\left(Y_{2} \mid U_{1}, U_{2}, Q\right) \\
& =H\left(Y_{2} \mid U_{1}, U_{2}, Q\right)+\theta I\left(Y_{2} ; U_{2} \mid U_{1}, Q\right) \\
& =H\left(Y_{2} \mid U_{1}, U_{2}, Q\right)+D_{1}-H\left(Y_{1} \mid U_{1}, Q\right)-I\left(Y_{2} ; U_{2} \mid Y_{1}, Q\right)+I\left(Y_{2} ; U_{2} \mid U_{1}, Q\right) \\
& =H\left(Y_{2} \mid U_{1}, U_{2}, Q\right)+D_{1}-H\left(Y_{1} \mid U_{1}, U_{2}, Q\right) \\
& \leq D_{2},
\end{aligned}
$$

where 68) follows from (55).

Thus, this proves that the Berger-Tung compression scheme can achieve any rate distortion tuple $\left(r_{1}, r_{2}, D_{1}, D_{2}\right)$ for $\left(r_{1}, r_{2}\right) \in \mathcal{P}$. Since $\mathcal{R} \mathcal{D}^{i}$ is, by definition, the set of rate distortion tuples attainable by the Berger-Tung achievability scheme, we must have that $\left(R_{1}, R_{2}, D_{1}, D_{2}\right) \in \mathcal{R D}^{i}$. This proves the lemma.

\section{F A Lemma for the Daily Double}

For a given joint distribution $p\left(y_{1}, y_{2}\right)$ on the finite alphabet $\mathcal{Y}_{1} \times \mathcal{Y}_{2}$, let $\mathcal{P}\left(R_{1}, R_{2}\right)$ denote the set of joint pmf's of the form

$$
p\left(q, y_{1}, y_{2}, u_{1}, u_{2}\right)=p(q) p\left(y_{1}, y_{2}\right) p\left(u_{1} \mid y_{1}, q\right) p\left(u_{1} \mid y_{1}, q\right)
$$

which satisfy

$$
\begin{aligned}
R_{1} & \geq I\left(Y_{1} ; U_{1} \mid U_{2}, Q\right) \\
R_{2} & \geq I\left(Y_{2} ; U_{2} \mid U_{1}, Q\right) \\
R_{1}+R_{2} & \geq I\left(Y_{1}, Y_{2} ; U_{1}, U_{2} \mid Q\right)
\end{aligned}
$$

for given finite alphabets $\mathcal{U}_{1}, \mathcal{U}_{2}, \mathcal{Q}$.

Lemma 10. For $R_{1}, R_{2}$ satisfying $R_{1} \leq H\left(Y_{1}\right), R_{2} \leq H\left(Y_{2}\right)$, and $R_{1}+R_{2} \leq$ $H\left(Y_{1}, Y_{2}\right)$, the infimum

$$
\inf _{p \in \mathcal{P}\left(R_{1}, R_{2}\right)}\left\{H\left(Y_{1} \mid U_{1}, U_{2}, Q\right)+H\left(Y_{2} \mid U_{1}, U_{2}, Q\right)\right\}
$$

is attained by some $p^{*} \in \mathcal{P}\left(R_{1}, R_{2}\right)$ which satisfies $R_{1}+R_{2}=$ $I\left(Y_{1}, Y_{2} ; U_{1}^{*}, U_{2}^{*} \mid Q^{*}\right)$, where $U_{1}^{*}, U_{2}^{*}, Q^{*}$ correspond to the auxiliary random variables defined by $p^{*}$. 
Proof. First, note that the infimum is always attained since $\mathcal{P}\left(R_{1}, R_{2}\right)$ is compact and the objective function is continuous on $\mathcal{P}\left(R_{1}, R_{2}\right)$. Therefore, let $U_{1}^{*}, U_{2}^{*}, Q^{*}$ correspond to the auxiliary random variables which attain the infimum.

If $H\left(Y_{1} \mid U_{1}^{*}, U_{2}^{*}, Q^{*}\right)+H\left(Y_{2} \mid U_{1}^{*}, U_{2}^{*}, Q^{*}\right)=0$, then we must have $I\left(Y_{1}, Y_{2} ; U_{1}^{*}, U_{2}^{*} \mid Q^{*}\right)=H\left(Y_{1}, Y_{2}\right)$. Thus, $R_{1}+R_{2}=I\left(Y_{1}, Y_{2} ; U_{1}^{*}, U_{2}^{*} \mid Q^{*}\right)$.

Next, consider the case where $H\left(Y_{1} \mid U_{1}^{*}, U_{2}^{*}, Q^{*}\right)+H\left(Y_{2} \mid U_{1}^{*}, U_{2}^{*}, Q^{*}\right)>0$. Assume for sake of contradiction that $R_{1}+R_{2}>I\left(Y_{1}, Y_{2} ; U_{1}^{*}, U_{2}^{*} \mid Q^{*}\right)$. For any $p \in \mathcal{P}\left(R_{1}, R_{2}\right)$ :

$$
I\left(Y_{1} ; U_{1} \mid U_{2}, Q\right)+I\left(Y_{2} ; U_{2} \mid U_{1}, Q\right) \leq I\left(Y_{1}, Y_{2} ; U_{1}, U_{2} \mid Q\right) .
$$

Hence, at most one of the remaining rate constraints can be satisfied with equality. If none of the rate constraints are satisfied with equality, then define

$$
\left(\tilde{U}_{1}, \tilde{U}_{2}\right)= \begin{cases}\left(U_{1}^{*}, U_{2}^{*}\right) & \text { with probability } 1-\epsilon \\ \left(Y_{1}, Y_{2}\right) & \text { with probability } \epsilon\end{cases}
$$

For $\epsilon>0$ sufficiently small, the distribution $\tilde{p}$ corresponding to the auxiliary random variables $\tilde{U}_{1}, \tilde{U}_{2}, Q^{*}$ is still in $\mathcal{P}\left(R_{1}, R_{2}\right)$. However, $\tilde{p}$ satisfies

$$
H\left(Y_{1} \mid \tilde{U}_{1}, \tilde{U}_{2}, Q^{*}\right)+H\left(Y_{2} \mid \tilde{U}_{1}, \tilde{U}_{2}, Q^{*}\right)<H\left(Y_{1} \mid U_{1}^{*}, U_{2}^{*}, Q^{*}\right)+H\left(Y_{2} \mid U_{1}^{*}, U_{2}^{*}, Q^{*}\right),
$$

which contradicts the optimality of $p^{*}$.

Therefore, assume without loss of generality that

$$
\begin{gathered}
R_{1}=I\left(Y_{1} ; U_{1}^{*} \mid U_{2}^{*}, Q^{*}\right) \\
R_{1}+R_{2}>I\left(Y_{1}, Y_{2} ; U_{1}^{*}, U_{2}^{*} \mid Q^{*}\right) .
\end{gathered}
$$

This implies that $R_{2}>I\left(Y_{2} ; U_{2}^{*} \mid Q^{*}\right)$. Now, define

$$
\tilde{U}_{2}= \begin{cases}U_{2}^{*} & \text { with probability } 1-\epsilon \\ Y_{2} & \text { with probability } \epsilon .\end{cases}
$$

Note that for $\epsilon>0$ sufficiently small:

$$
\begin{aligned}
I\left(Y_{2} ; U_{2}^{*} \mid Q^{*}\right) & <I\left(Y_{2} ; \tilde{U}_{2} \mid Q^{*}\right)<R_{2} \\
I\left(Y_{1}, Y_{2} ; U_{1}^{*}, U_{2}^{*} \mid Q^{*}\right) & <I\left(Y_{1}, Y_{2} ; U_{1}^{*}, \tilde{U}_{2} \mid Q^{*}\right)<R_{1}+R_{2},
\end{aligned}
$$

and for any $\epsilon \in[0,1]$ :

$$
\begin{aligned}
R_{1}=I\left(Y_{1} ; U_{1}^{*} \mid U_{2}^{*}, Q^{*}\right) & \geq I\left(Y_{1} ; U_{1}^{*} \mid \tilde{U}_{2}, Q^{*}\right) \\
H\left(Y_{1} \mid U_{1}^{*}, U_{2}^{*}, Q^{*}\right)+H\left(Y_{2} \mid U_{1}^{*}, U_{2}^{*}, Q^{*}\right) & \geq H\left(Y_{1} \mid U_{1}^{*}, \tilde{U}_{2}, Q^{*}\right)+H\left(Y_{2} \mid U_{1}^{*}, \tilde{U}_{2}, Q^{*}\right) .
\end{aligned}
$$

Since $R_{2} \leq H\left(Y_{2}\right)$, as $\epsilon$ is increased from 0 to 1 , at least one of the following must occur: 
1. $I\left(Y_{2} ; \tilde{U}_{2} \mid Q^{*}\right)=R_{2}$.

2. $I\left(Y_{1}, Y_{2} ; U_{1}^{*}, \tilde{U}_{2} \mid Q^{*}\right)=R_{1}+R_{2}$.

3. $I\left(Y_{1} ; U_{1} \mid \tilde{U}_{2}, Q^{*}\right)<R_{1}$.

If either of events 1 or 2 occur first then the sum-rate constraint is met with equality (since they are equivalent in this case). If event 3 occurs first, then all rate constraints are satisfied with strict inequality and we can apply the above argument to contradict optimality of $p^{*}$. Since 69 ) shows that the objective is nonincreasing in $\epsilon$, there must exist a $\tilde{p} \in \mathcal{P}\left(R_{1}, R_{2}\right)$ which attains the infimum and satisfies the sum-rate constraint with equality.

\section{References}

[1] D. Slepian and J. Wolf, "Noiseless coding of correlated information sources," Information Theory, IEEE Transactions on, vol. 19, pp. 471 480, Jul 1973.

[2] R. Ahlswede and J. Korner, "Source coding with side information and a converse for degraded broadcast channels," Information Theory, IEEE Transactions on, vol. 21, pp. 629 - 637, Nov 1975.

[3] A. Wyner, "On source coding with side information at the decoder," Information Theory, IEEE Transactions on, vol. 21, pp. 294 - 300, May 1975.

[4] A. Wyner and J. Ziv, "The rate-distortion function for source coding with side information at the decoder," Information Theory, IEEE Transactions on, vol. 22, pp. 1 - 10, Jan. 1976.

[5] T. Berger and R. Yeung, "Multiterminal source encoding with one distortion criterion," Information Theory, IEEE Transactions on, vol. 35, pp. 228 -236, Mar 1989.

[6] A. Wagner, S. Tavildar, and P. Viswanath, "Rate region of the quadratic gaussian two-encoder source-coding problem," Information Theory, IEEE Transactions on, vol. 54, pp. 1938 -1961, May 2008.

[7] V. Prabhakaran, D. Tse, and K. Ramachandran, "Rate region of the quadratic gaussian ceo problem," in Information Theory, 2004. ISIT 2004. Proceedings. International Symposium on, p. 119, june-2 july 2004.

[8] Y. Oohama, "Gaussian multiterminal source coding," Information Theory, IEEE Transactions on, vol. 43, pp. 1912 -1923, nov 1997.

[9] N. Cesa-Bianchi and G. Lugosi, Prediction, Learning, and Games. New York, NY, USA: Cambridge University Press, 2006. 
[10] T. Courtade and R. Wesel, "Multiterminal source coding with an entropybased distortion measure," in Information Theory Proceedings (ISIT), 2011 IEEE International Symposium on, pp. 2040 -2044, Aug. 2011.

[11] P. Harremoes and N. Tishby, "The information bottleneck revisited or how to choose a good distortion measure," in Information Theory, 200\%. ISIT 2007. IEEE International Symposium on, pp. 566 -570, June 2007.

[12] T. Andre, M. Antonini, M. Barlaud, and R. Gray, "Entropy-based distortion measure for image coding," in Image Processing, 2006 IEEE International Conference on, pp. 1157 -1160, Oct. 2006.

[13] T. Berger, Z. Zhang, and H. Viswanathan, "The ceo problem [multiterminal source coding]," Information Theory, IEEE Transactions on, vol. 42, pp. 887 -902, may 1996.

[14] T. Berger, Multiterminal Source Coding. In G. Longo (Ed.), The Information Theory Approach to Communications. New York, NY, USA: SpringerVerlag, 1977.

[15] S.-Y. Tung, Multiterminal Source Coding. PhD thesis, Cornell University, Ithaca, NY, 1978.

[16] A. El Gamal and Y.-H. Kim, Network Information Theory. Cambridge University Press, 2012.

[17] S. Jana, "Alphabet sizes of auxiliary random variables in canonical inner bounds," in Information Sciences and Systems, 2009. CISS 2009. 43rd Annual Conference on, pp. 67 -71, March 2009.

[18] T. M. Cover and J. A. Thomas, Elements of Information Theory. John Wiley \& Sons, 2nd ed., 2006.

[19] E. Erkip, The Efficiency of Information in Investment. PhD thesis, Stanford University, 1996.

[20] Y.-H. Kim, A. Sutivong, and T. Cover, "State amplification," Information Theory, IEEE Transactions on, vol. 54, pp. 1850 -1859, may 2008.

[21] J. Korner and K. Marton, "How to encode the modulo-two sum of binary sources (corresp.)," Information Theory, IEEE Transactions on, vol. 25, pp. 219 - 221, Mar 1979.

[22] W. Gu and M. Effros, "On approximating the rate region for source coding with coded side information," in Information Theory Workshop, 200\%. ITW '07. IEEE, pp. $432-435$, Sept. 2007.

[23] W. Gu, S. Jana, and M. Effros, "On approximating the rate regions for lossy source coding with coded and uncoded side information," in Information Theory, 2008. ISIT 2008. IEEE International Symposium on, pp. 2162 2166, July 2008. 
[24] H. Witsenhausen, "Some aspects of convexity useful in information theory," Information Theory, IEEE Transactions on, vol. 26, pp. 265 - 271, May 1980.

[25] A. Schrijver, Combinatorial Optimization: Polyhedra and Efficiency. Berlin: Springer-Verlag, 2003.

[26] S. Fujishige, Submodular Functions and Optimization. Berlin: Elsevier Science, 2nd ed., 2010.

[27] S. McCormick, Submodular Function Minimization. In Discrete Optimization, K. Aardal, G. Nemhauser, and R. Weismantel, eds. Handbooks in Operations Research and Management Science, vol. 12. Elsevier, 2005. 